\title{
Signature of Shallow Potentials in Deep Sub-barrier Fusion Reactions
}

\author{
Ş. Mişicu* and H. Esbensen \\ Physics Division, Argonne National Laboratory, Argonne, Illinois 60439, USA
}

(Dated: August 7, 2021)

\begin{abstract}
We extend a recent study that explained the steep falloff in the fusion cross section at energies far below the Coulomb barrier for the symmetric dinuclear system ${ }^{64} \mathrm{Ni}+{ }^{64} \mathrm{Ni}$ to another symmetric system, ${ }^{58} \mathrm{Ni}+{ }^{58} \mathrm{Ni}$, and the asymmetric system ${ }^{64} \mathrm{Ni}+{ }^{100} \mathrm{Mo}$. In this scheme the very sensitive dependence of the internal part of the nuclear potential on the nuclear equation of state determines a reduction of the classically allowed region for overlapping configurations and consequently a decrease in the fusion cross sections at bombarding energies far below the barrier. Within the coupled-channels method, including couplings to the low-lying $2^{+}$and $3^{-}$states in both target and projectile as well as mutual and two-phonon excitations of these states, we calculate and compare with the experimental fusion cross sections, $S$-factors, and logarithmic derivatives for the above mentioned systems and find good agreement with the data even at the lowest energies. We predict, in particular, a distinct double peaking in the $S$-factor for the far subbarrier fusion of ${ }^{58} \mathrm{Ni}+{ }^{58} \mathrm{Ni}$ which should be tested experimentally.
\end{abstract}

PACS numbers: 24.10.Eq, 25.60.-t, 25.60.Pj, 25.70.-z, 25.70.Jj

\footnotetext{
* on leave of absence from National Institute for Nuclear Physics, Bucharest, P.O.Box MG6, Romania.
} 


\section{INTRODUCTION}

Very recently we proposed a mechanism that can explain the unexpected hindrance or steep falloff of fusion cross sections which has been observed at bombarding energies far below the Coulomb barrier [1]. Although measurements of several medium-heavy nuclei performed in the past two decades already provided some indications of a steep decrease of the excitation functions at the lowest bombarding energies, the credit of disclosing and confirming this unexpected trend for new fusing systems has to be given to C. L. Jiang et al. [2, 3, 4 4, 5, 6]. Among the most conspicuous cases reported in the past are ${ }^{58} \mathrm{Ni}+{ }^{58} \mathrm{Ni}$ [7], where the departure from the expected behavior takes places already at cross sections $\sim 0.1$ $\mathrm{mb}$, and ${ }^{90} \mathrm{Zr}+{ }^{89} \mathrm{Y},{ }^{90} \mathrm{Zr}+{ }^{92} \mathrm{Zr}$ as reported in [8] ]

The new fusion data reported by Jiang et al. are even more spectacular because the reported cross sections are measured down to $10 \mathrm{nb}:{ }^{60} \mathrm{Ni}+{ }^{89} \mathrm{Y}[2]\left(\sigma_{f} \geq 100 \mathrm{nb}\right),{ }^{64} \mathrm{Ni}+$ ${ }^{64} \mathrm{Ni}$ [4] $\left(\sigma_{f} \geq 10 \mathrm{nb}\right),{ }^{64} \mathrm{Ni}+{ }^{100} \mathrm{Mo} \mathrm{[5]}\left(\sigma_{f}>10 \mathrm{nb}\right)$. The hindrance of fusion was first reported as a suppression of the measured low-energy fusion cross sections with respect to model calculations [2]. It was later on characterized by the energy $E_{s}$ where the $S$-factor for fusion develops a maximum at low energy [3]. Following the publication of these findings a challenge was launched, especially on the theoretical side of the heavy-ion fusion community. At the end of 2005 the underlying physical cause of this apparently new phenomenon was still unknown according to the authors of Ref. [6]. Some authors have even advocated the hypothesis that the standard theoretical approach to treat capture reactions, i. e. the coupled-channels (C. C.) method is unable to explain the steep falloff of the cross sections. We shall try to convince the reader throughout this paper that the C. C. method is the right tool to investigate capture, even at very low energy.

The nuclear potentials that are employed in C. C. calculations are commonly parametrized as a Woods-Saxon well. Among the issues related to the deep sub-barrier fusion was the large diffuseness $a$ of the ion-ion potential that was needed to fit high-precision fusion data. Hagino et al. [9], hinted that a phenomenological nuclear potential with a larger diffuseness leads to a better agreement to the data. Values up to $a=1.3 \mathrm{fm}$ were conjectured instead of the usual $a=0.65 \mathrm{fm}$ for the system ${ }^{58} \mathrm{Ni}+{ }^{58} \mathrm{Ni}$. In [3] it was remarked that since the low-energy fusion becomes sensitive to the nuclear potential inside the barrier, this part of the interaction may not be accurately modeled by the conventional Woods-Saxon 
parametrization. It was pointed out that by doubling the diffuseness of the inner part of the potential, the agreement with the data improves for the colliding system ${ }^{60} \mathrm{Ni}+{ }^{89} \mathrm{Y}[3]$.

Following the same idea, the systematic failure of the Woods-Saxon nuclear potential to describe fusion was analyzed in [10] and it was concluded that the origin of it should be sought in the diffuseness parameter $a$. In order to fit the data at energies above the Coulomb barrier, the diffuseness must be increased with increasing $Z_{1} Z_{2}$. The fusion data compiled by these authors indicate a correlation with the neutron richness of the projectile and target nuclei in the sense that the neutron rich nuclei tend to require larger values of $a$.

The authors of [11] pointed out that potentials such as the Akyüz-Winther (AW) [12, 13] are providing reliable barriers but they cannot reproduce the data far below the barrier, a fact which made them suggest that the ion-ion potential should have another form in the inner part of the barrier. Following a sequence of simple but clear arguments they pointed out that the exponential falloff in the tunneling probability is related to the disappearance of the classically allowed region below a certain energy. If this is true, it would mean that we are confronted with the existence of a shallow pocket of the potential inside the barrier.

In Ref. [14] a surprisingly good description of the data for ${ }^{58} \mathrm{Ni}+{ }^{58} \mathrm{Ni},{ }^{64} \mathrm{Ni}+{ }^{64} \mathrm{Ni}$ and ${ }^{60} \mathrm{Ni}+{ }^{89} \mathrm{Y}$ was claimed. However, it is difficult to judge the significance of the results because of the limited number of excitations that were included in the C. C. calculations. For example, the only excitation considered in the nickel isotopes is the one-phonon $2^{+}$excitation, but it is well known that couplings to the $3^{-}$state and higher-order couplings to two-phonon states are tremendously enhancing the heavy-ion fusion cross section [15, 16].

Other works have attempted to account for the complicated problem of channel coupling by means of a polarization potential [17]. The imaginary potential parametrizes in this case the excitation of other degrees of freedom that influence the fusion process. According to [17], the imaginary potential shows a rapid cutoff at energies far below the Coulomb barrier for the cases ${ }^{58} \mathrm{Ni}+{ }^{58} \mathrm{Ni},{ }^{58} \mathrm{Ni}+{ }^{64} \mathrm{Ni}$ and ${ }^{64} \mathrm{Ni}+{ }^{64} \mathrm{Ni}$ because of a threshold below which the C. C. effects are ceasing to exist.

Before ending the list of hypotheses advanced to explain the fusion hindrance phenomenon we quote the result reported in Ref. [18], which overrules the possibility of explaining the depletion of fusion rates at extreme sub-barrier energies from dissipative tunneling, as results from other quantum open system approaches, such as that of Caldeira and Leggett [19].

Very recently [1] we proposed an explanation of the hindrance observed in the sub-barrier 
fusion of ${ }^{64} \mathrm{Ni}+{ }^{64} \mathrm{Ni}$, which was based on the same standard coupled-channels formalism as before [3] but with amendments that concern the potential. Essential in getting a good description of the data was to take into account the saturation of nuclear matter and to use realistic neutron and proton distributions of the reacting nuclei. These two ingredients are naturally incorporated in a potential calculated via the double-folding method with tested effective nucleon-nucleon forces and with realistic charge and nuclear densities, a fact which is often overlooked or only indirectly included in the Woods-Saxon parametrization. We intend in this paper to show that by properly addressing these issues, light can be shed on the extreme sub-barrier fusion data. Before we do that we summarize the methods that have been used to analyze the low-energy behavior of heavy-ion fusion cross sections.

\section{REPRESENTATIONS OF LOW-ENERGY CROSS SECTIONS}

In an attempt to get a diagnosis for the various cases where the hindrance in sub-barrier fusion occurs, the authors of Ref. [3] proposed the use of two representations. The first one is the astrophysical $S$-factor [20],

$$
S=E \sigma(E) \exp (2 \pi \eta)
$$

where $E$ is the center-of-mass energy, $\eta=Z_{1} Z_{2} e^{2} /\left(\hbar v_{\text {rel }}\right)$ is the Sommerfeld parameter and $v_{\text {rel }}$ is the relative velocity of the fragments. The experimental value of $S$ increases with decreasing bombarding energy and has the tendency to develop a maximum for the systems of interest. The necessity to resort to this quantity comes from the fact that the reaction cross section varies by many orders of magnitude below the Coulomb barrier (7 orders of magnitude for ${ }^{64} \mathrm{Ni}+{ }^{64} \mathrm{Ni}$ ). The $S$-factor has been used in the past to unravel typical molecular resonant structures in the excitation function of systems like ${ }^{12} \mathrm{C}+{ }^{12} \mathrm{C}$ since it removes the dominating influence of the Coulomb and centrifugal barrier transmission factors that mask these structures in the cross section [21]. The series of narrow and prominent resonances was associated with quasi-bound, long-lived states of the ${ }^{24} \mathrm{Mg}$ nucleus.

Very recently the fusion cross section for the ${ }^{12} \mathrm{C}+{ }^{12} \mathrm{C}$ system has been measured down to very low energies [22]. The data show a rise in the $S$-factor at the lowest energies, which might indicate the existence of a broad resonance in the entrance channel, possibly related to an intermediate state in the compound nucleus [22]. Similar broad resonances in the 
$S$-factor at energies below the barrier have been also inferred from the ${ }^{12} \mathrm{C}+{ }^{16} \mathrm{O}$ total cross sections [23]. Thus, the $S$-factor is a quantity that magnifies structures in the excitation function at energies below the barrier, and it is also an instrument for exploring the inner part of the barrier in low-energy, heavy-ion fusion reactions.

A second representation proposed in Ref. [3] is the logarithmic derivative,

$$
L(E)=\frac{d[\ln (E \sigma)]}{d E}=\frac{1}{E \sigma} \frac{d}{d E}(E \sigma) .
$$

The point where the experimental $L(E)$ intersects the logarithmic derivative obtained from an $s$-wave transmission across a pure point-charge Coulomb potential (constant $S$-factor), given by $L_{0}(E)=\frac{\pi \eta}{E}$, coincides with the maximum in the $S$-factor invoked earlier. Extracting the energy $E_{s}$, where this intersection occurs, it was found that the corresponding logarithmic derivative $L_{s}$ is nearly the same for stiff heavy-ion systems, with an average value of 2.34 $\mathrm{MeV}^{-1}$. This implies that $E_{s}$ scales with the charge and mass numbers of the reacting nuclei according to the empirical law [3]

$$
E_{s}=0.355\left[Z_{1} Z_{2} \sqrt{\frac{A_{1} A_{2}}{A_{1}+A_{2}}}\right]^{2 / 3} .
$$

At this point it is useful to recall that the inability of previous calculations to reproduce the low-energy data points of the measured fusion cross sections is most clearly seen from the inspection of the logarithmic derivative $L(E)$. Thus for energies below a certain threshold, the experimental values of $L(E)$ increase steeply with decreasing energy, whereas the theoretical curve increases with a much smaller slope on which a resonant structure is superposed.

\section{SIMULATIONS OF A REPULSIVE CORE}

The occurrence of resonant structures in collisions of light nuclei, the best known example being the sharp peaks in the bombarding energy dependence of the gamma radiation yields emerging in the ${ }^{12} \mathrm{C}+{ }^{12} \mathrm{C}$ scattering found by Bromley et al. [24], were found to resemble states in a molecular potential well. Following the suggestion that these "quasi-molecular" states may represent doorway states to fusion, the resonant behavior found its explanation by introducing the concept of the double-resonance mechanism [25]. In this scenario the indirect population of quasi-molecular states can occur in light-ion scattering according to 
the following sequence of events: a) Surpassing the potential barrier at an initial energy $E_{i}$ and losing the energy $E^{*}$ by inelastic excitations of low-energy levels of one or both of the ions, and b) Resonant decay into the potential pocket, where the colliding nuclei are trapped and a quasi-molecule is formed, if their relative energy $E_{i}-E^{*}$ coincides with the energy of a quasi-bound state.

Evidence that the resonant behavior observed in ${ }^{12} \mathrm{C}+{ }^{12} \mathrm{C}$ is not an isolated phenomenon was made available for other light systems such as ${ }^{12} \mathrm{C}+{ }^{16} \mathrm{O},{ }^{12} \mathrm{C}+{ }^{13} \mathrm{C},{ }^{16} \mathrm{O}+{ }^{16} \mathrm{O},{ }^{16} \mathrm{O}+$ ${ }^{24} \mathrm{Mg}$. It persists even in heavier colliding systems, such as the ${ }^{24} \mathrm{Mg}+{ }^{24} \mathrm{Mg}$ [26] and ${ }^{28} \mathrm{Si}+$ ${ }^{28} \mathrm{Si}$ [27]. Manifestation of clusterization in connection to quasi-molecular pockets is also known for heavy nuclear systems, such as the cluster radioactivity [28], or hyperdeformation and clustering in the actinide region [29, 30].

To simulate the appearance of shallow pockets several recipes have been proposed :

- A central soft repulsive core added to the conventional Woods-Saxon potential was used in Refs. [31] and [32] in order to fit the reaction cross sections observed in ${ }^{12} \mathrm{C}+$ ${ }^{12} \mathrm{C},{ }^{12} \mathrm{C}+{ }^{16} \mathrm{O}$ and ${ }^{16} \mathrm{O}+{ }^{16} \mathrm{O}$. The effect of a repulsive core was modeled by a positive Gaussian potential, $V_{\text {rep }} \exp \left(-b r^{2}\right)$, the width $b$ being constrained by the requirement that the potential becomes repulsive for $r \leq R_{0}, R_{0}$ being the radius of the WoodsSaxon potential.

- Double-folding potentials as introduced by Satchler and Love [33] are accurate only in the tail region of the nucleus-nucleus potential, where the density distributions are only gently overlapping and thus the assumption of "frozen density" is less questionable. However this assumption ignores any readjustment due to the mutual interaction of the nuclei or the Pauli exclusion principle for strong overlap. To cope with this problem the observation was made in [34, 35] that, whereas any theory of heavy-ion potentials is expected to reduce to the double-folding model in the limit of large ion-ion separations and vanishing density overlap, the compound system resulting from fusing the two ions is accurately described by the liquid-drop energy $E_{\mathrm{LDM}}$. To interpolate between these two extremes the nuclear deformation energy was written as

$$
E_{\mathrm{def}}=E_{\mathrm{LDM}}+W_{M 3 Y+C}(\rho)-\alpha \bar{W}_{M 3 Y}(\bar{\rho}),
$$

where $W_{M 3 Y+C}(\rho)$ is the double folding self-interaction energy with the original M3Y parametrization for the nuclear and Coulomb forces with realistic values of the proton 
and neutron matter diffusivities and radii. The $\bar{W}_{M 3 Y}(\bar{\rho})$ is a renormalization introduced with the purpose to subtract the volume and surface energy contributions in $W$, and is computed with sharp-edge densities. The parameter $\alpha$ is adjusted so that $W$ and $\bar{W}$ cancel exactly in the limit of complete overlap. This scheme was applied only for collisions of two identical spherical nuclei since the evaluation becomes to cumbersome for deformed nuclei approaching at different orientations.

- Pockets in a double folding potential are arising naturally if an effective Skyrme like $N N$ force is used [36]. However, this force was not tested systematically for scattering reactions as is the case for the M3Y force.

- Density-dependent interactions superposed on the original M3Y form have been used in recent times to simulate the saturation of nuclear matter for $\alpha+$ nucleus scattering or elastic scattering of light nuclei [37, 38]. An attempt to explain fusion data for a variety of systems using these density dependent interactions was made very recently by the Canberra group [39] and they reached the conclusion that the double-folding model may not be appropriate for fusion. The pockets resulting from the density dependence of the effective $N N$ force are still too deep to improve the agreement of C. C. calculations and the experimental data at extreme sub-barrier energies.

- Methods based on the energy density functional are known for a long time to be able to predict shallow pockets in the interaction between two nuclei [40, 41]. A typical feature of this class of potentials is the short-ranged repulsion at distances where a strong overlap of the nuclear densities takes place. In this approach the condition of nuclear matter saturation is achieved by fitting the free parameters in the energy-density functional using the properties of finite nuclei. Like in the case of the double-folding recipe, the sudden-approximation, i.e. the summation of frozen nuclear densities, is essential for the occurrence of the core. Very recently, using this method, in the framework of the extended Thomas-Fermi approximation with $\hbar^{2}$ correction terms in the kinetic energy density functional, an analytic potential with parameters that are fitted to data was proposed [42]. However, this potential provides barriers that are higher than the experimental ones and they can therefore not provide a satisfactory description of the data in the barrier region. 
- Proximity potentials are well-known examples of nuclear interactions producing pockets in the ion-ion potential (see Ref. [43] for the 1977 and [44] for the 2000 versions). Unfortunately these potentials provide pockets that are too deep for the systems of interests (see for example Fig. 1 of Ref. [1]).

- Finally we mention the semi-empirical Akyüz-Winther potential which we will refer to in the following as the AW potential. It is parametrized as a Woods-Saxon potential [12], so that the maximum nuclear force is consistent with the proximity force for touching spheres, and it has an exponential tail that is consistent with the M3Y double-folding calculated in Ref. [13]. The AW also produces pockets that are too deep as evidenced by its applications in Refs. [1, 2, 3, 4, 5, 6].

In the present work we are interested in the interaction between two intermediate mass nuclei. For these target-projectile combinations the above approach to the density dependent interaction does not necessarily apply because, as we shall see, the resulting pockets are still to deep. For this reason another approach will be adopted.

\section{THE M3Y+REPULSION, DOUBLE-FOLDING POTENTIAL}

As a manifestation of the Pauli principle, which prevents the overlapping of the wave functions of two systems of fermions, we expect that the interaction potential between two colliding nuclei will contain a repulsive core. The actual form of the repulsive core and its strength depend strongly on the extent to which the collision is adiabatic or sudden. A further uncertainty with regard to the core parameters is the influence of individual characteristics of the considered nuclei, including binding energies, shapes, and the nucleon distributions. The models of ion-ion potentials which provide a repulsive core and which we enumerated in the preceding section, lead to quantitatively different estimates of the height, radius and diffuseness of the core potential. Due to these conditions of strong uncertainty we use like in previous works on fusion [45] a crude recipe to determine the properties of the repulsive core potential. 


\section{A. Calibration of the repulsive core potential}

As noticed earlier, an overlapping region with doubled nucleon density is formed once the distance $r$ between the nuclei becomes less than $R_{1}+R_{2}$, where $R_{1}$ and $R_{2}$ are the nuclear radii of the target and projectile along the scattering (fission) axis. Here and in the following we assume for simplicity that the densities of the two nuclei are frozen when estimating the nuclear interaction potential. When a complete overlap of the two nuclei occurs, the total density is therefore roughly twice that of normal matter, $\rho \approx 2 \rho_{0}$, within the volume of the smaller nucleus. Consequently, the nuclear equation-of-state (EOS) dictates an increase $\Delta V$ in the energy of the compound system. In the case of complete overlap (for $r=0$ ) $\Delta V$ is proportional to the increase of the energy per particle of nuclear matter $\varepsilon(\rho, \delta)$ considered as a function of the nuclear density, $\rho=\rho_{n}+\rho_{p}$, and the relative neutron excess, $\delta=\left(\rho_{n}-\rho_{p}\right) / \rho$,

$$
\Delta V \approx 2 A_{p}\left[\varepsilon\left(2 \rho_{0}, \delta\right)-\varepsilon\left(\rho_{0}, \delta\right)\right]
$$

Here the relative neutron excess $\delta$ is assumed to be approximately constant, and $A_{p}$ is the mass number of the smaller nucleus in the case of an asymmetric system.

The EOS predicted by the Thomas-Fermi model for cold nuclear matter is [46]

$$
\varepsilon(\rho, \delta)=\varepsilon_{F}\left[A(\delta)\left(\frac{\rho}{\rho_{0}}\right)^{2 / 3}+B(\delta)\left(\frac{\rho}{\rho_{0}}\right)+C(\delta)\left(\frac{\rho}{\rho_{0}}\right)^{5 / 3}\right],
$$

where $\rho_{0}=0.161 \mathrm{fm}^{-3}$ is the saturation density and $\varepsilon_{F}$ is the Fermi energy of normal nuclear matter. The expressions for the $\delta$-dependence entering Eq. (66) are listed in Ref. [46]. The definition of the incompressibility of cold nuclear matter is then related to the curvature of $\varepsilon(\rho, \delta)$ at the saturation density,

$$
K=9\left(\rho^{2} \frac{\partial^{2} \varepsilon}{\partial \rho^{2}}\right)_{\rho=\rho_{0}} .
$$

In order to calibrate the strength of the repulsive core potential, we assume that $\Delta V$ in Eq. (5) must be identified with the nuclear part of the heavy-ion potential, $V_{N}(r)$, evaluated at the coordinate origin $r=0$,

$$
\Delta V=V_{N}(0)
$$

Approximating the EOS by a parabolic expansion around the equilibrium density $\rho_{0}$ [47],

$$
\varepsilon(\rho, \delta)=\varepsilon\left(\rho_{0}, \delta\right)+\frac{K}{18 \rho_{0}^{2}}\left(\rho-\rho_{0}\right)^{2}
$$


we obtain the following estimate of the nuclear potential at $r=0$,

$$
V_{N}(0)=\Delta V \approx \frac{A_{p}}{9} K
$$

Similar recipes for introducing a repulsive core can be found in the literature [48]. They are based on the knowledge of the equation of state and on the requirement that the nuclear matter density is doubled for a total overlap of the two reacting nuclei.

\section{B. Folding Model Potential}

We consider two nuclei with one-body deformed densities $\rho_{1}$ and $\rho_{2}$, subjected to vibrational fluctuations, and center of masses separated by the distance $\boldsymbol{r}$. Then the nuclear potential between these two nuclei can be evaluated as the double folding integral,

$$
V_{N}(\boldsymbol{r})=\int d \boldsymbol{r}_{1} \int d \boldsymbol{r}_{2} \rho_{1}\left(\boldsymbol{r}_{1}\right) \rho_{2}\left(\boldsymbol{r}_{2}\right) v\left(\boldsymbol{r}_{12}\right),
$$

where $\boldsymbol{r}_{12}=\boldsymbol{r}+\boldsymbol{r}_{\mathbf{2}}-\boldsymbol{r}_{1}$. The central part of the effective $N N$ interaction $v\left(\boldsymbol{r}_{12}\right)$ contains no spin or spin-isospin terms since we analyze fusion reactions of spin 0 nuclei, where the spin terms are relatively unimportant. We are then left with a direct part,

$$
v_{\text {dir }}\left(\boldsymbol{r}_{12}\right)=v_{00}\left(\boldsymbol{r}_{12}\right)+\frac{N_{1}-Z_{1}}{A_{1}} \frac{N_{2}-Z_{2}}{A_{2}} v_{01}\left(\boldsymbol{r}_{12}\right),
$$

which depends on isospin since $N \neq Z$ in all the cases of interest. The exchange part, which takes into account the effect of antisymmetrization under the exchange of nucleons between the two nuclei, is modeled by the contact interaction,

$$
v_{\mathrm{ex}}\left(\boldsymbol{r}_{12}\right)=\left(\hat{J}_{00}+\frac{N_{1}-Z_{1}}{A_{1}} \frac{N_{2}-Z_{2}}{A_{2}} \hat{J}_{01}\right) \delta\left(\boldsymbol{r}_{12}\right) .
$$

The density independent part of the effective nucleon-nucleon force that we use is the Reid parametrization of Michigan-3-Yukawa (M3Y) interaction [49]. The explicit form of the expressions for $v_{00}, v_{01}, \hat{J}_{00}$ and $\hat{J}_{01}$ are given, for example, in Ref. [50]. Moreover, we neglect the possible energy dependence of these parameters. For example, a variation $\delta E \approx 25$ $\mathrm{MeV}$ between the minimum and maximum bombarding energies considered in the fusion of ${ }^{64} \mathrm{Ni}+{ }^{64} \mathrm{Ni}$ gives a variation of $\delta \hat{J}_{00} \approx 0.27 \mathrm{MeV} \cdot \mathrm{fm}^{3} \mathrm{MeV}$ in the Reid parametrization, a values that is small compared to $\hat{J}_{00} \approx-276 \mathrm{MeV} \cdot \mathrm{fm}^{3}$.

As we noted earlier, a potential that is based on the M3Y interaction predicts correctly the ion-ion potential for peripheral collisions. However, reactions that are sensitive to the 


\begin{tabular}{|c|c|c|c|c|}
\hline Nucleus & $r_{0 p}(\mathrm{fm})$ & $a_{p}(\mathrm{fm})$ & $r_{0 n}(\mathrm{fm})$ & $a_{n}(\mathrm{fm})$ \\
\hline${ }^{58} \mathrm{Ni}$ & 1.107 & 0.4673 & 1.0836 & 0.5124 \\
\hline${ }^{64} \mathrm{Ni}$ & 1.0652 & 0.575 & 1.0852 & 0.532 \\
\hline${ }^{100} \mathrm{Mo}$ & 1.1025 & 0.535 & 1.105 & 0.575 \\
\hline
\end{tabular}

TABLE I: Parameters of the proton and neutron density distributions, Eq. (15), which have been used in the calculation of the direct and exchange, double-folding potential.

potential at smaller distances are not reproduced [37]. To cure this deficiency, the ionion potential is supplemented with a short-ranged repulsive potential which, according to the discussion in the previous subsection, is proportional to the overlapping volume of the reacting nuclei. This is simulated in Eq. (11) by using the effective contact interaction,

$$
v_{\text {rep }}\left(\boldsymbol{r}_{12}\right)=V_{\text {rep }} \delta\left(\boldsymbol{r}_{12}\right)
$$

We follow the procedure proposed in Ref. [48] and use a relatively sharp density profile, characterized by the diffuseness $a_{\text {rep }}$, when calculating the repulsive potential from the double-folding integral, Eq. (11). The strength $V_{\text {rep }}$ of the repulsive interaction is then obtained from the condition Eq. (10), which relates the total nuclear potential at the coordinate origin to the nuclear incompressibility.

\section{Parametrization of densities}

The densities that appear in Eq. (11) are parametrized with Fermi-Dirac distribution functions

$$
\rho_{i}(\boldsymbol{r})=\frac{\rho_{0}}{1+\exp \left[\left(r-R_{0 i}\right) / a\right]}
$$

where $R_{0 i}=r_{0 i} A_{i}^{1 / 3}$. The parameters of the proton and neutron density distributions we have used are listed in Table $\mathbb{I}$. The parameters for the proton density of ${ }^{64} \mathrm{Ni}$ are taken from a compilation of elastic electron scattering data [51]. For the neutron distribution we choose the parameters such that they are in the range of what one would expect for a moderately neutron-rich nucleus, and such that the barrier of the M3Y+repulsion potential is close to the one predicted by the Akyüz-Winther potential, since this potential gives a good description of the data in the barrier and high-energy region [4]. The parameters 


\begin{tabular}{|c|c|c|c|c|}
\hline Reaction & $a_{\text {rep }}(\mathrm{fm})$ & $V_{\text {rep }}\left(\mathrm{MeV} \cdot \mathrm{fm}^{3}\right)$ & $K(\mathrm{MeV})$ & $V_{\text {pocket }}(\mathrm{MeV})$ \\
\hline${ }^{58} \mathrm{Ni}+{ }^{58} \mathrm{Ni}$ & 0.341 & 510.1 & 234 & 89.31 \\
\hline${ }^{64} \mathrm{Ni}+{ }^{64} \mathrm{Ni}$ & 0.405 & 511.0 & 228 & 84.98 \\
\hline${ }^{64} \mathrm{Ni}+{ }^{100} \mathrm{Mo}$ & 0.375 & 488.7 & 226 & 117.8 \\
\hline
\end{tabular}

TABLE II: Parameters used in the calculation of the repulsive, double-folding potential for three fusion reactions, and the associated incompressibility and pocket energy.

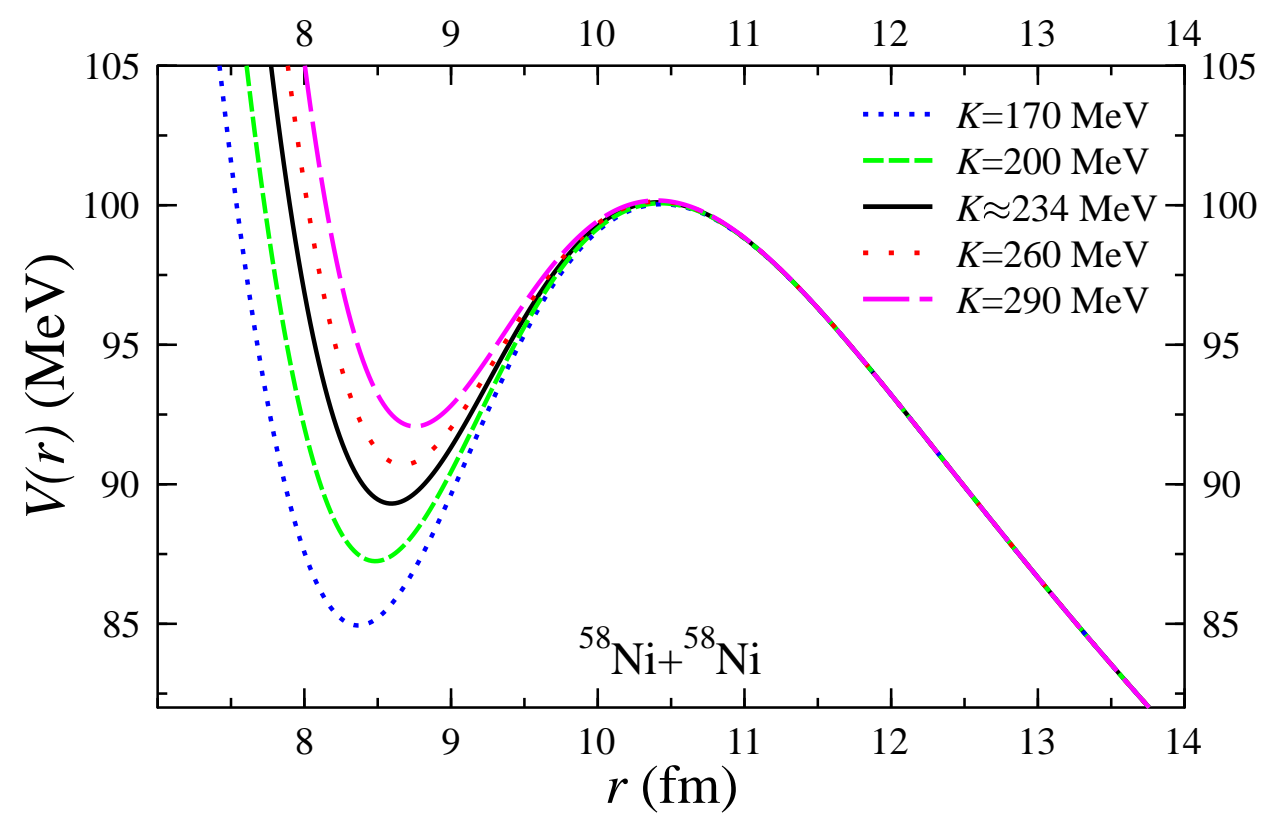

FIG. 1: (Color online). Coulomb plus the nuclear M3Y+repulsion, double-folding potential for ${ }^{58} \mathrm{Ni}+{ }^{58} \mathrm{Ni}$. Results are shown for different values of the nuclear incompressibility $K$.

for the proton and neutron densities in ${ }^{58} \mathrm{Ni}$ and ${ }^{100} \mathrm{Mo}$ we have used are identical or close to those predicted by the Hartree-Fock-Bogoliubov (HFB) method using the BSk2 Skyrme force [52].

\section{Heavy-ion potential study cases}

Applying the formalism described in subsections A and B, we vary the parameters $a_{\text {rep }}$ and $V_{\text {rep }}$ of the repulsive potential so that Eq. (10) is approximately fulfilled for the nuclear incompressibility $K$ predicted by the Thomas-Fermi model [46]. The parameters we obtain 


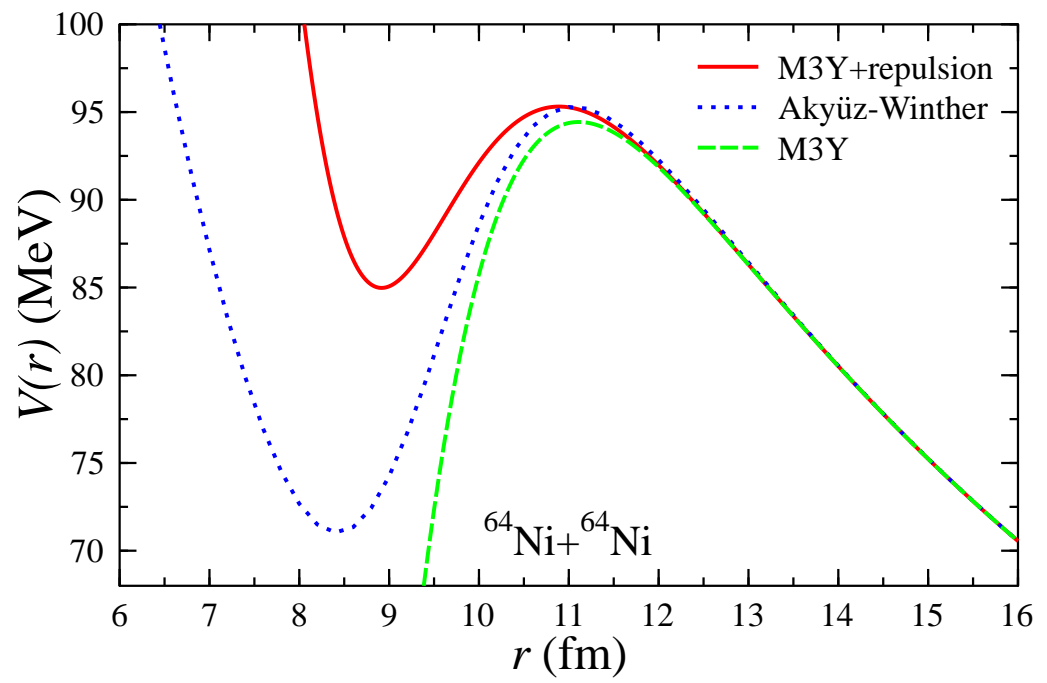

FIG. 2: (Color online). Comparison of the double folding potentials with (solid curve) and without (long-dashed curve) repulsion, and the Akyüz-Winther potential (short dashed curve) for the system ${ }^{64} \mathrm{Ni}+{ }^{64} \mathrm{Ni}$.

for the three heavy-ion systems we have studied are listed in Table II, together with the incompressibility of the compound nuclei obtained in the Thomas-Fermi model, and the energy of the resulting pocket that appears in the Coulomb plus nuclear potential.

To illustrate the dependence of the total ion-ion potential on the nuclear equation of state we plot in Fig. 1 the Coulomb plus nuclear potential for the dinuclear system ${ }^{58} \mathrm{Ni}+{ }^{58} \mathrm{Ni}$ for different choices of the nuclear incompressibility $K$. The solid black curve corresponds to the incompressibility $K \approx 234 \mathrm{MeV}$ as inferred from the Thomas-Fermi model for ${ }^{116} \mathrm{Ba}$. The effect of $K$ on the inner part of the barrier and the depth of the pocket is essential. A soft EOS provides a deep pocket whereas a hard one results in a shallow pocket.

The effect of the repulsive core is displayed in Fig. 2, We compare the total potentials obtained from standard M3Y heavy-ion potential, the M3Y+repulsion potential for ${ }^{64} \mathrm{Ni}+{ }^{64} \mathrm{Ni}$, and the the Akyüz-Winther, for the system ${ }^{64} \mathrm{Ni}+{ }^{64} \mathrm{Ni}$. Due to the lower barrier and an abrupt decrease in the inner region, the M3Y potential cannot accurately reproduce the data, a fact that already has been pointed out in the literature [10, 39].

In Fig. 3 we show the profiles of the Coulomb plus nuclear potentials for four heavy-ion systems. The solid curves are based on the M3Y+repulsion potentials we use in this work, whereas the short-dashed curves are based on the AW potential. It is seen that the pockets 

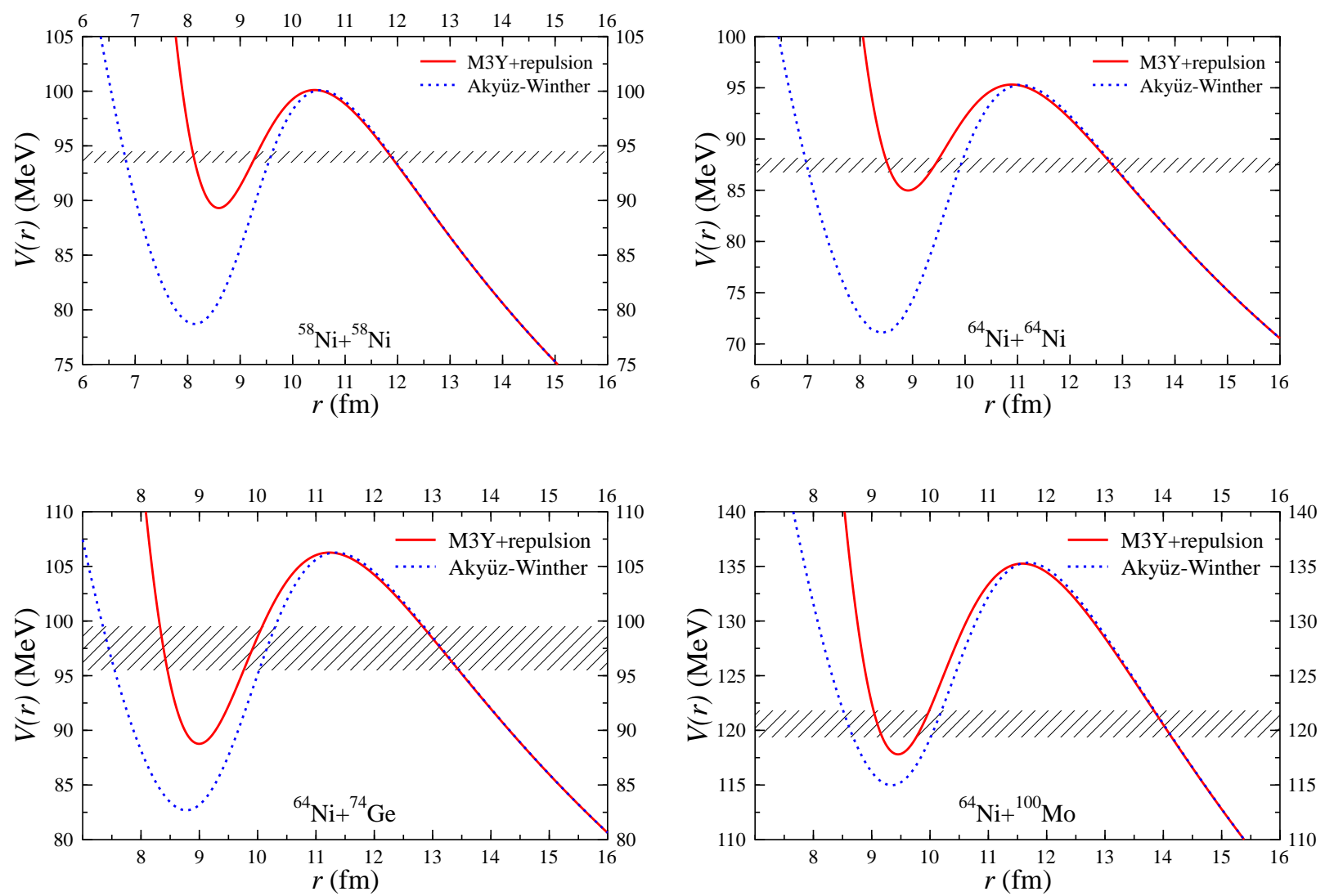

FIG. 3: (Color online). Ion-ion potentials for ${ }^{58} \mathrm{Ni}+{ }^{58} \mathrm{Ni},{ }^{64} \mathrm{Ni}+{ }^{64} \mathrm{Ni},{ }^{64} \mathrm{Ni}+{ }^{74} \mathrm{Ge},{ }^{64} \mathrm{Ni}+{ }^{100} \mathrm{Mo}$. The solid curves are the potentials employed in the present work. The short-dashed curves are based on the Akyüz-Winther potential, which was used in Refs. [2, 3, 4, 5, 흐. The dashed strips show for each system the experimental boundaries of the threshold energy $E_{s}[6]$.

predicted for the M3Y+repulsion potentials are much shallower than predicted by the AW potential, in particular for the lighter systems. The dashed region in Fig. 3 shows the energy $E_{s}$ where the experimental $S$-factor develops a maximum, and the width of it illustrates the uncertainty in the extracted value of $E_{s}[6]$.

\section{COUPLED-CHANNELS APPROACH}

We use the same approach as in previous publications (see [53] and references therein), i.e. coupled-channels calculations performed in the so-called iso-centrifugal or rotating-frame approximation, where it is assumed that the orbital angular momentum $L$ for the relative 
motion of the dinuclear system is conserved. The rotating frame approximation allows a drastic reduction of the number of channels used in the calculations.

The only ingredient we are going to change in the formalism is the ion-ion potential. Like in the case of other nuclear potentials we resort to the "proximity" approximation [12] which states that the heavy-ion potential is a function of the shortest distance between the nuclear surfaces of the reacting nuclei. Since for spherical nuclei, the relative orientation is not coming into play, the fragments have the natural tendency to be polarized along the collision axis, which is defined by the radial separation vector $\boldsymbol{r}$. Surface vibrations are then taking place parallel (along) the direction $\boldsymbol{r}$. In this case the shortest distance between the two surfaces is

$$
s=r-R_{1}-R_{2}-\delta R,
$$

where

$$
\delta R=R_{1} \sum_{\lambda \mu} \alpha_{\lambda \mu}^{(1)} Y_{\lambda \mu}^{*}(\hat{r})+R_{2} \sum_{\lambda \mu} \alpha_{\lambda \mu}^{(2)} Y_{\lambda \mu}^{*}(-\hat{r}),
$$

and $\hat{r}$ specifies the spatial orientation of the dinuclear system in the laboratory system and $\alpha_{\lambda \mu}^{(i)}$ are the deformation parameters. In the rotating frame approximation, which we will use, the direction of $\boldsymbol{r}$ defines the $z$-axis. The only vibrational excitations that can take place are therefore the $\mu=0$ components, since $Y_{\lambda \mu}(\hat{z}) \propto \delta_{\mu, 0}$.

The cases under study in this paper refer only to the generation of vibrational excitations of surface modes. In previous papers it was shown that the inclusion of linear and quadratic interactions is necessary and often sufficient to fit the data at least in the intermediate energy region (see [53] and references therein). It is then necessary to consider only the spherical part of the double-folding potential, and its first and second order derivatives. The nuclear potential for the elastic channel is then given by Eq. (11), which is most conveniently calculated by the Fourier transform [54],

$$
V(r)=\frac{1}{2 \pi^{2}} \int d q q^{2} \rho_{1}(q) \rho_{2}(q) v(q) j_{0}(q r)
$$

Here $\rho_{i}(q)$ is the Fourier transform of the spherical ground state density of ion $i, v(q)$ the Fourier transform of the effective $N N$ interactions, and $j_{0}(q r)$ stands for the spherical Bessel function. This is actually the potential we have already shown in Figs. 1-3.

The non-spherical part of the nuclear potential that results from the difference between the total interaction and the potential in the elastic channel is expanded up to second-order 
in the surface distortion (17),

$$
\delta V_{N}(r)=-\frac{\partial V}{\partial r} \delta R+\frac{1}{2} \frac{\partial^{2} V}{\partial r^{2}}\left[(\delta R)^{2}-\left\langle 0\left|(\delta R)^{2}\right| 0\right\rangle\right]
$$

It is seen that the ground state expectation of this interaction, $\left\langle 0\left|\delta V_{N}\right| 0\right\rangle$, is zero, but the second order term will give a non-zero contribution to the diagonal matrix element in an excited state. One can show that this prescription is exact for a harmonic oscillator up to second order in the deformation amplitudes. We include a similar expansion of the Coulomb field, $\delta V_{C}$, but only to first order in the deformation amplitudes [53]. These expressions are inserted into the C. C. formalism in the rotating frame approximation which singles out only axially symmetric distortions $\left(\alpha_{\lambda \mu=0}\right)$, i.e.,

$$
\begin{gathered}
\left(\frac{\hbar^{2}}{2 M_{0}}\left[-\frac{d^{2}}{d r^{2}}+\frac{L(L+1)}{r^{2}}\right]+\frac{Z_{1} Z_{2} e^{2}}{r}+V(r)+\sum_{n_{1}, n_{2}} \varepsilon_{n_{1}, n_{2}}-E\right) u_{n_{1} n_{2}}(r) \\
=-\sum_{m_{1} m_{2}}\left\langle n_{1} n_{2}\left|\delta V_{C}+\delta V_{N}\right| m_{1} m_{2}\right\rangle u_{m_{1} m_{2}}(r),
\end{gathered}
$$

where $E$ is the relative energy in the center of mass frame, $L$ is the conserved orbital angular momentum, and $M_{0}$ is the reduced mass of the dinuclear system. The C. C. equations (20) are written for two coupled vibrators of eigenenergy $\varepsilon_{n_{1}, n_{2}}$ and consequently the radial wave function $u(r)$ is labeled by the quantum numbers $n_{1}$ and $n_{2}$. Expressions for the matrix elements of $\delta V$ in the double-oscillator basis are given in [15].

The above C. C. equations are solved with the usual boundary conditions at large distances and appropriate in-going-wave boundary conditions imposed inside the barrier, more precisely, at the radial separation where the potential pocket attains its minimum. The fusion cross section results then from the total in-going flux. The calculations include one-phonon excitations of the lowest $2^{+}$and $3^{-}$states in target and projectile, and all two-phonon and mutual excitations of these states up to a 7.2 MeV excitation energy. This energy cutoff was chosen so that all of the two-phonon states were included in the calculations for ${ }^{64} \mathrm{Ni}+{ }^{64} \mathrm{Ni}$ and ${ }^{64} \mathrm{Ni}+{ }^{100} \mathrm{Mo}$, whereas the two-phonon octupole states were excluded in the calculations for ${ }^{58} \mathrm{Ni}+{ }^{58} \mathrm{Ni}$. The necessary structure input for ${ }^{64} \mathrm{Ni}$ and ${ }^{100} \mathrm{Mo}$ is given in Ref. [55]; the input for ${ }^{58} \mathrm{Ni}$ is from Ref. [15]. 


\section{CROSS-SECTIONS, $S$-FACTORS AND LOGARITHMIC DERIVATIVES}

In what follows we analyze the fusion data for three systems for which there are strong indications of a hindrance, -or steep falloff of the cross sections, at energies well below the Coulomb barrier. For completeness we also show in section VI.4 the high-energy behavior of the cross sections. All calculations are based on the coupled-channels formalism outlined in the previous section, and further interpretations of the results are presented in Sect. VII.

\section{1. ${ }^{58} \mathrm{Ni}+{ }^{58} \mathrm{Ni}$}

The first case we consider is the fusion reaction of ${ }^{58} \mathrm{Ni}+{ }^{58} \mathrm{Ni}$. For this system data are available from an older experiment and the smallest cross sections are in the range of $\mathrm{mb}$ [7]. The data are compared in Fig. 4 to several C. C. calculations that are based on the same structure input as in Ref. [15] and on the M3Y+repulsion potential, but they differ in the assumed value of the nuclear incompressibility. We see that for a hard nuclear EOS $(K=290 \mathrm{MeV})$ the calculated low-energy cross sections deviate strongly from the measured values. Obviously in this case the origin of the mismatch is primarily due to the existence of a very shallow pocket which reduces the classically allowed region and hinders the fusion drastically. For a soft nuclear $\operatorname{EOS}(K=170 \mathrm{MeV})$ the potential pocket is lowered and the calculated cross sections approach the results calculated with the Akyüz-Winther potential. The best fit to the data is obtained for the nuclear incompressibility $K=234 \mathrm{MeV}$, which is the value one obtains in the extended Thomas-Fermi model.

Some of the results shown Fig. 4 are repeated in Fig. 5, where they are compared to similar results for the fusion of ${ }^{64} \mathrm{Ni}+{ }^{64} \mathrm{Ni}$ and ${ }^{64} \mathrm{Ni}+{ }^{100} \mathrm{Mo}$, which will be discussed in more detail below. The C. C. calculations for the M3Y+repulsion potential (solid curves) were all performed with the nuclear incompressibility derived from the extended Thomas-Fermi model. The long-dashed curves are the no-coupling limits (NOC) obtained with the AW potential.

In Fig. 6 (left panel) we compare the experimental $S$-factors for ${ }^{58} \mathrm{Ni}+{ }^{58} \mathrm{Ni}$ to the those calculated with the M3Y+repulsion and the AW potentials. In the last case the mismatch with the data is already evident starting at energies $4-5 \mathrm{MeV}$ below the barrier. The former case, on the other hand, provides a good description of the available data and, most im- 


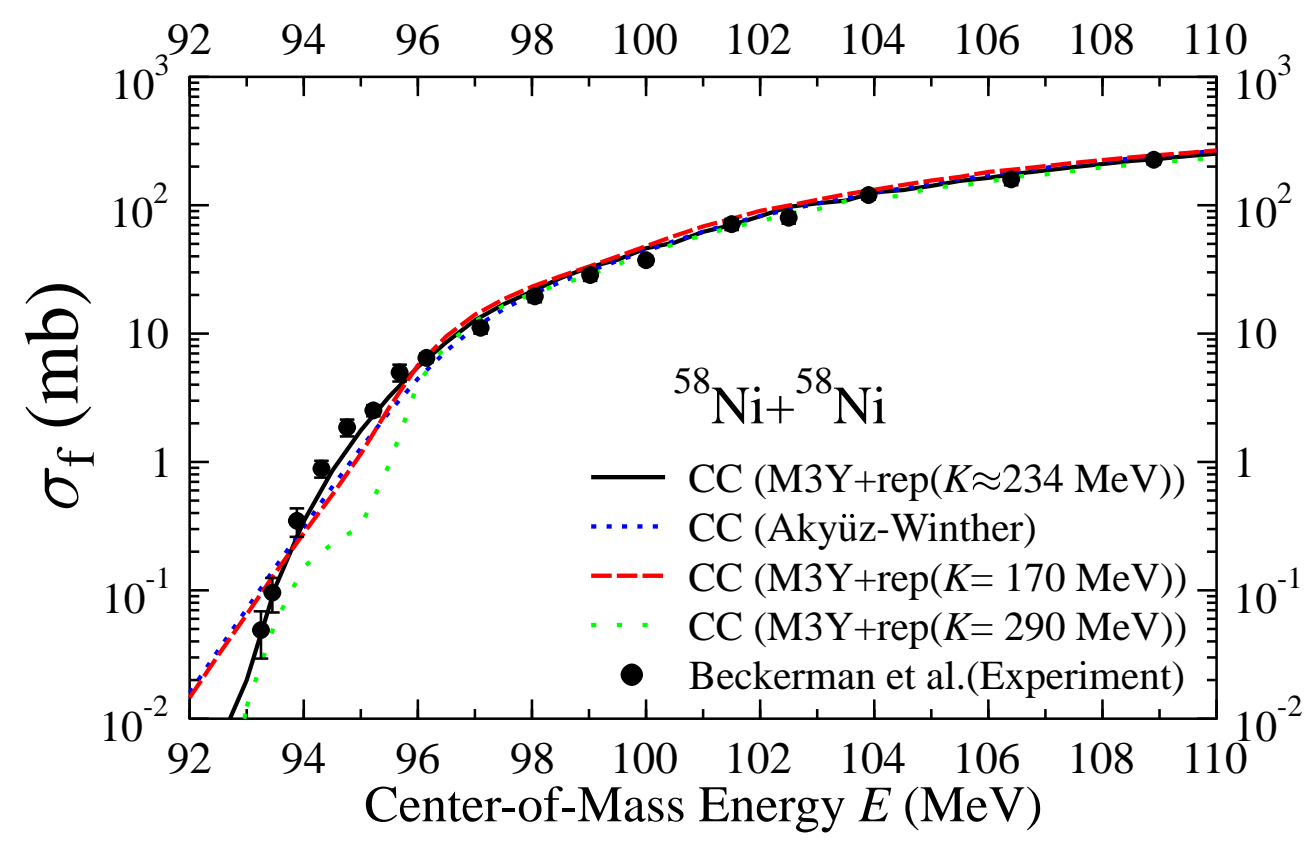

FIG. 4: (Color online). Experimental fusion excitation function for the system ${ }^{58} \mathrm{Ni}+{ }^{58} \mathrm{Ni}$ [7] is compared to various C. C. calculations that are based on the M3Y+repulsion potential (assuming different values of the nuclear incompressibility $K$ ), and on the AW potential.

portantly, reproduces the trend of the experimental $S$-factor to produce a maximum. The calculation also predicts a second maximum at even lower energies where no data exist. It remains to be seen whether future measurements will confirm the two predicted maxima.

In Fig. 7 (lower panel) we plot the logarithmic derivative of the energy-weighted cross section for the case ${ }^{58} \mathrm{Ni}+{ }^{58} \mathrm{Ni}$. We obtain a nice description of the available experimental data when we use the M3Y+repulsion potential (solid curve). As expected from the behavior of the $S$-factor, $L(E)$ produces a maximum (just above the constant $S$-factor limit) followed by a local minimum, before it finally diverges at lower energies. To ascertain this behavior, and also the predicted excitation function and $S$-factor for ${ }^{58} \mathrm{Ni}+{ }^{58} \mathrm{Ni}$, high-precision fusion data are necessary at very low bombarding energies,

\section{2. ${ }^{64} \mathrm{Ni}+{ }^{64} \mathrm{Ni}$}

Next we consider the system ${ }^{64} \mathrm{Ni}+{ }^{64} \mathrm{Ni}$. This case was already discussed in our previous publication [1] but for the sake of completeness we find it necessary to recall it and add more 

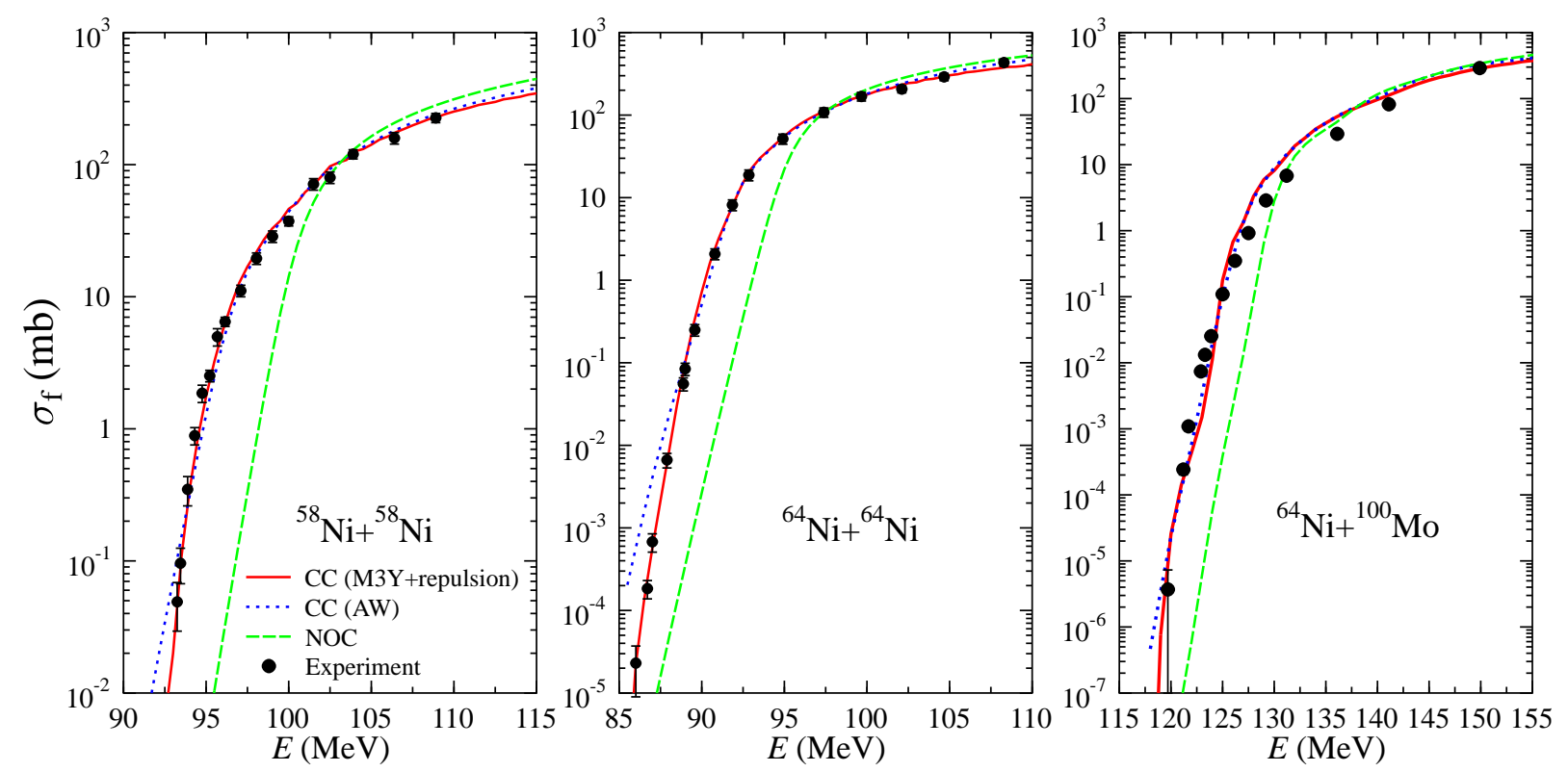

FIG. 5: (Color online). Experimental fusion excitation functions for the systems ${ }^{58} \mathrm{Ni}+{ }^{58} \mathrm{Ni}$ [7], ${ }^{64} \mathrm{Ni}+{ }^{64} \mathrm{Ni}$ [4], and ${ }^{64} \mathrm{Ni}+{ }^{100} \mathrm{Mo}$ [5] are compared to various C. C. calculations described in the text, and to the no-coupling (NOC) limit for the AW potential.

features like, for example, the logarithmic derivative.

The system ${ }^{64} \mathrm{Ni}+{ }^{64} \mathrm{Ni}$ was advocated to be a very good choice for precise measurements since there is no contamination from reactions with heavier isotopes in the target, or from lower- $Z$ isobaric components in the beam, which can dominate the yield at extreme subbarrier energies [4]. The ATLAS data for this case are in a good agreement with previous results [56] at energies above the Coulomb barrier but not around and below the barrier. However, the agreement is good around and below the barrier with an older experiment [7]. It should be also mentioned that the ATLAS data provide cross sections down to tenths of nanobarns [3] compared to the $0.3 \mathrm{mb}$ that was reached in the older experiment [7].

To obtain the incompressibility of $K \approx 228 \mathrm{MeV}$ for total overlap of the reacting nuclei, as predicted by the Thomas-Fermi model, we use a strength of $V_{\text {rep }}=511 \mathrm{MeV}$ and a diffuseness of the repulsive part of the density distribution of $a_{\text {rep }} \approx 0.4 \mathrm{fm}$ for both protons and neutrons.

In Fig. 5 (middle panel) we compare the experimental excitation function for the fusion reaction ${ }^{64} \mathrm{Ni}+{ }^{64} \mathrm{Ni} \rightarrow{ }^{128} \mathrm{Ba}$ with the C. C. results obtained using the Akyüz-Winther potential like in Ref. [2] (dotted line) and with the M3Y+repulsion potential (solid line). 

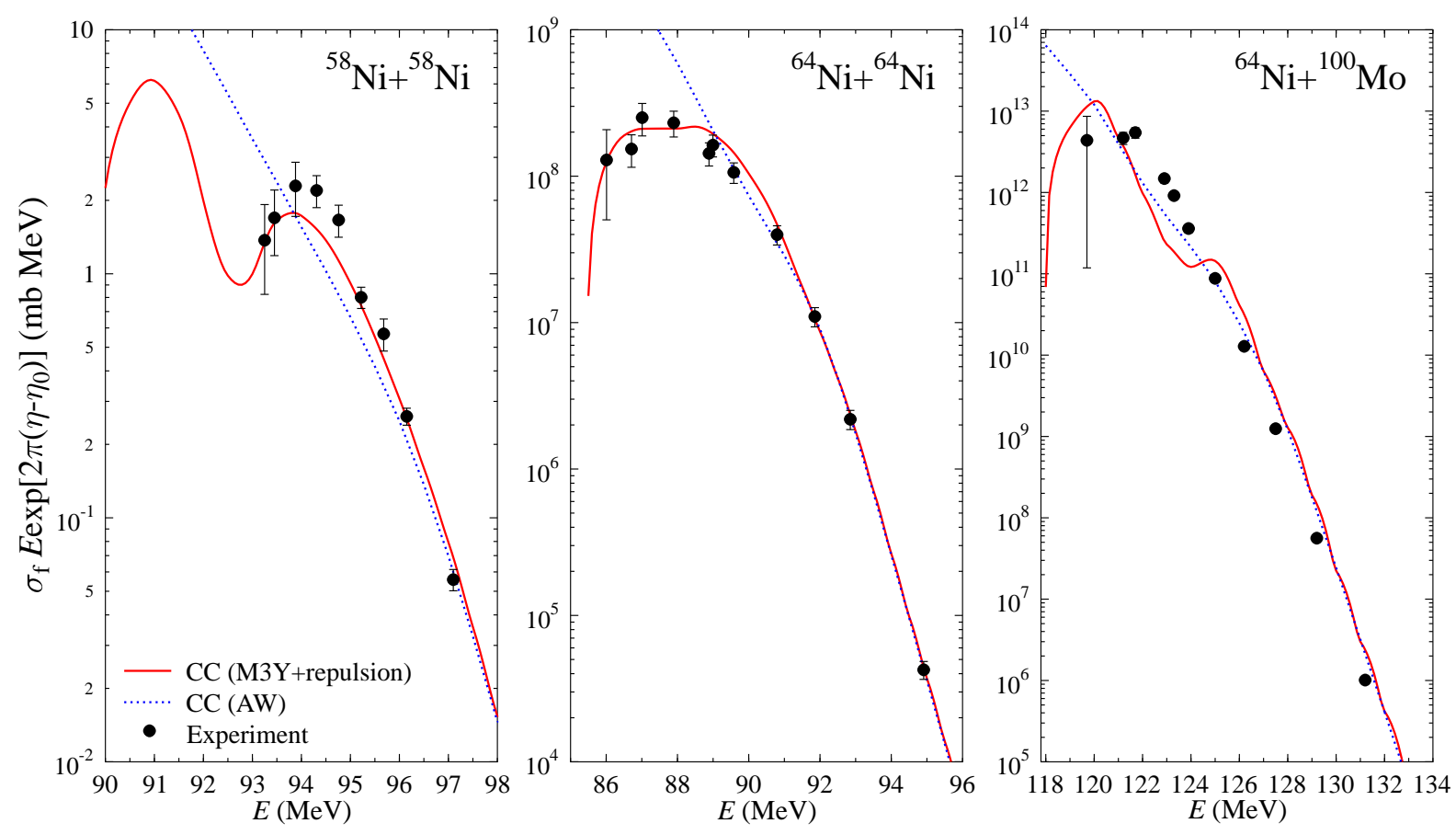

FIG. 6: (Color online). The experimental $S$-factors for the systems ${ }^{58} \mathrm{Ni}+{ }^{58} \mathrm{Ni}$ [7] (left panel), ${ }^{64} \mathrm{Ni}+{ }^{64} \mathrm{Ni}[4]$ (middle panel) and ${ }^{64} \mathrm{Ni}+{ }^{100} \mathrm{Mo}[\underline{5}$ ] (right panel) are indicated by solid circles. They are compared to the coupled-channels calculations performed with the M3Y+repulsion (solid curve) and Akyüz-Winther (dashed curve) potentials.

The same recipe for the C. C. calculations was used in both cases. The diffuseness of the Akyüz-Winther potential we use is $a=0.676 \mathrm{fm}$. One should also recall that calculations using a modified Akyüz-Winther potential, where the diffuseness inside the barrier was set to $a_{i}=5 \mathrm{fm}$, were not able to the date to explain the steep fall-off of the measured cross section [3]. This is not surprising because the modified AW potential has a very deep pocket (see Fig. 4 of Ref. [53] for an interior diffuseness of $a_{i}=10 \mathrm{fm}$.)

We conclude, from the inspection of Fig. 5 that the agreement with data, when using the $\mathrm{M} 3 \mathrm{Y}+$ repulsion potential, is sensitively better than the one provided by the Akyüz-Winther starting at $90 \mathrm{MeV}$, and not only for the 4 lowest experimental data points. The excitation function obtained with the M3Y+repulsion potential has the right slope, not only because the potential attains a higher-lying pocket but also because the curvature of the barrier is different, with a thicker barrier in the overlapping region. Thus the best $\chi^{2}$ per point is only $\chi^{2} / N=0.86$. This value is obtained by applying the energy shift $\Delta E=0.16 \mathrm{MeV}$ to the calculated excitation function. The best fit obtained with AW potential, on the other hand, 

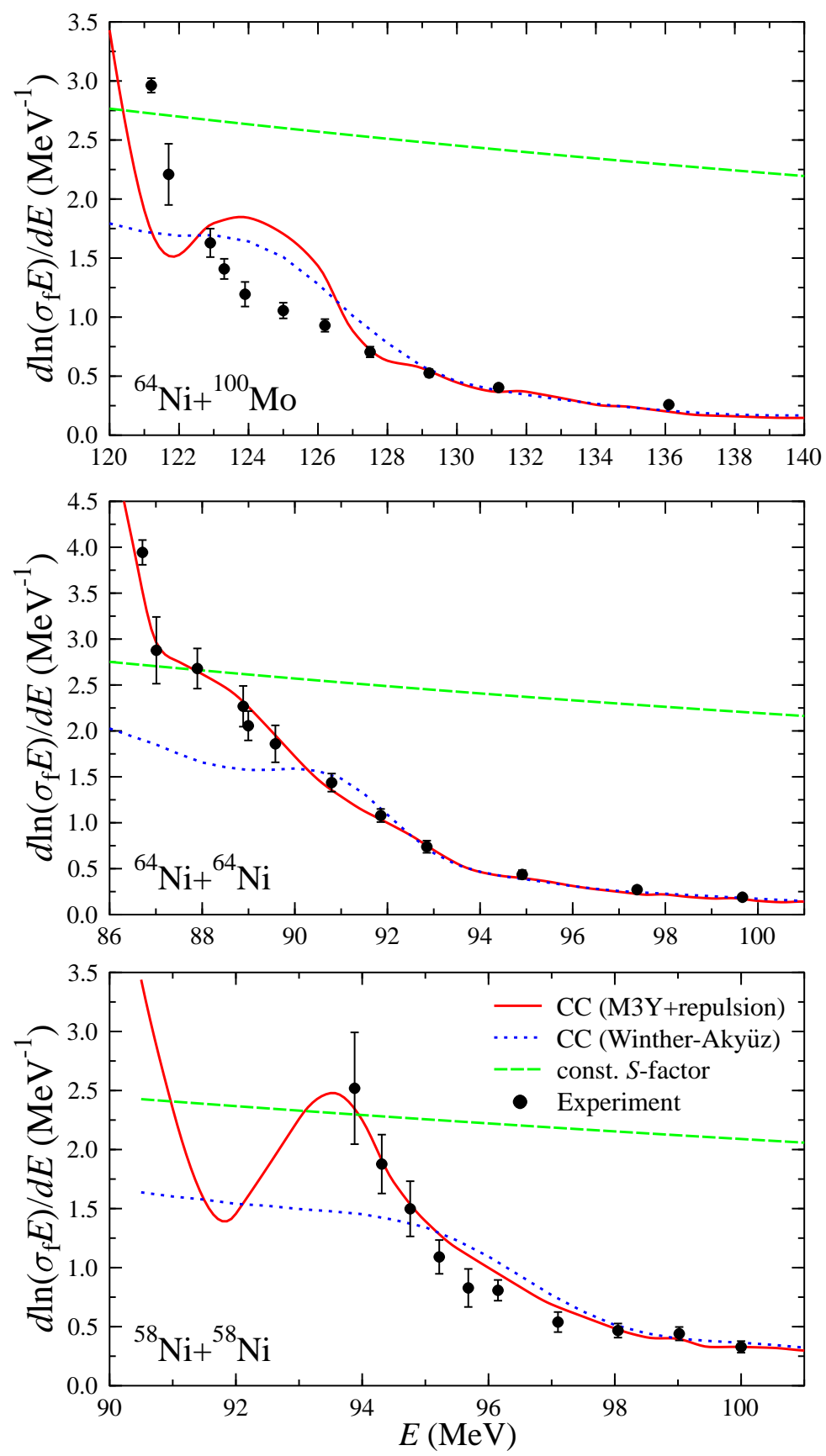

FIG. 7: (Color online). Logarithmic derivatives of the energy-weighted cross sections for ${ }^{58} \mathrm{Ni}+{ }^{58} \mathrm{Ni}$ (lower panel), ${ }^{64} \mathrm{Ni}+{ }^{64} \mathrm{Ni}$ (middle panel), and ${ }^{64} \mathrm{Ni}+{ }^{100} \mathrm{Mo}$ (upper panel). Experimental results derived from the cross sections shown in Fig. 5 are compared to coupled-channels calculations performed with the M3Y+repulsion (solid curve) and the Akyüz-Winther (dashed curve) potential. The top dashed curve in each panel is the prediction for a constant $S$-factor. 
gives a $\chi^{2} / N=10$ and requires an energy shift of $\Delta E=0.9 \mathrm{MeV}$.

The $S$-factor representation of the ${ }^{64} \mathrm{Ni}+{ }^{64} \mathrm{Ni}$ fusion cross sections is displayed in the middle panel of Fig. 6. The clear maximum in the measured $S$-factor is reproduced only by the M3Y+repulsion potential. At this point we recall the experience gained in the past on molecular resonances. As shown in Ref. [23], the $S$-factor exhibits a sequence of quasi-molecular resonances, sandwiched between a limiting interior threshold and the Coulomb barrier. In the present case we obtain a maximum that is too broad to be assigned to a resonance, the curvature in the $S$-factor being explained by the shallow pocket in the potential. The maximum of the theoretical curve corresponds approximately to the maximum of the experimental data.

The logarithmic derivative of the energy-weighted cross section is shown in the middle panel of Fig. 7, and a nice agreement with the data is obtained in the C. C. calculations that are based on the M3Y+repulsion potential.

$$
\text { 3. }{ }^{64} \mathrm{Ni}+{ }^{100} \mathrm{Mo}
$$

For the repulsive part of the potential we choose $a_{\text {rep }}=0.375 \mathrm{fm}$ and $V_{\text {rep }}=488.7 \mathrm{MeV}$, values that are matching the incompressibility $K=226 \mathrm{MeV}$ of the compound nucleus ${ }^{164} \mathrm{Yb}$. To improve the fit to the data we included up to three phonon excitations of the quadrupole mode in ${ }^{100}$ Mo using the structure parameters given in Ref. [55]. However, the agreement with the data seen in the right panel of Fig. 5 is clearly not as good as in the other two cases shown in the same figure. The reason is that the C. C. effects are very strong for this heavy-ion system and the calculations have not fully converged with respect to multiphonon excitations, as discussed in Ref. [55]. Another problem is that the nuclear structure properties of multiphonon states are often poorly known, so we will not try to improve the fit to the data here.

For the $S$-factor we reproduce roughly, as can be concluded from an inspection of the right panel in Fig. 6, the trend to develop a well pronounced maximum at the lowest measured energies and provide a reasonable estimation for this maximum. Also the logarithmic derivative manifests the tendency to develop a divergent behavior at low energies (Fig. 7 , upper panel). The appearance of a local maximum at $124 \mathrm{MeV}$, on the other hand, is most likely caused by a poor convergence with respect to multiphonon excitations. 


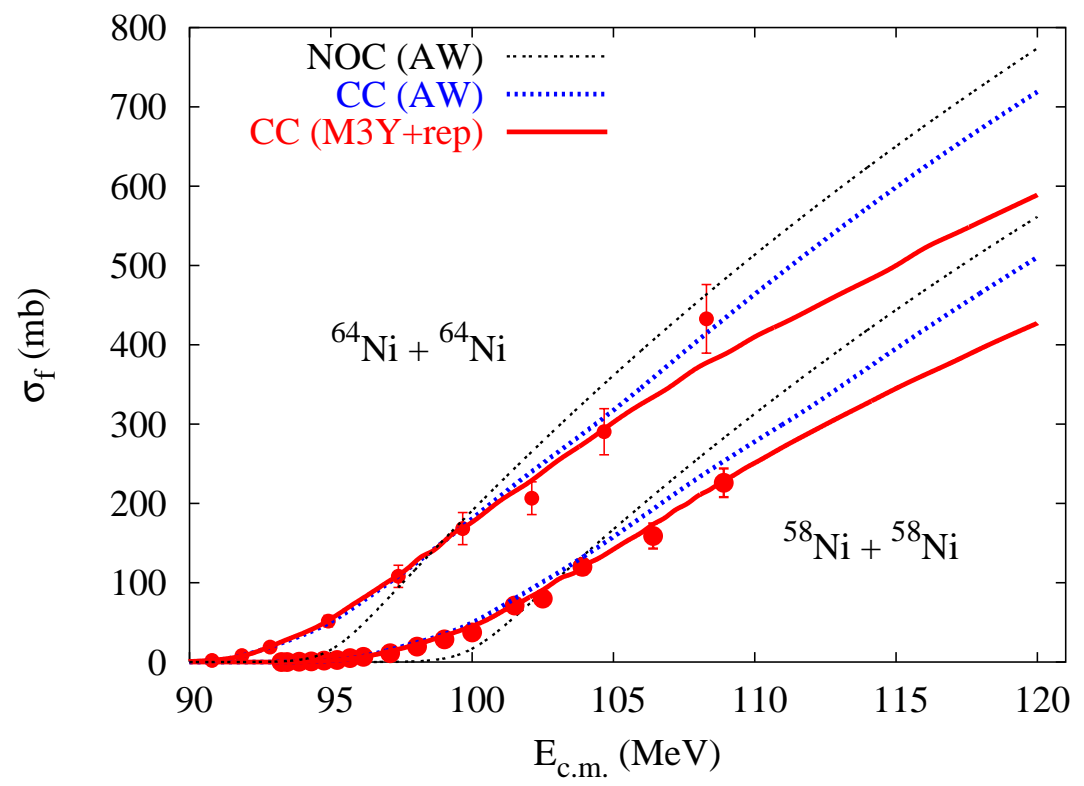

FIG. 8: (Color online). Linear plot of the fusion cross sections shown in Fig. 5 for the two systems ${ }^{64} \mathrm{Ni}+{ }^{64} \mathrm{Ni}[4]$ and ${ }^{58} \mathrm{Ni}+{ }^{58} \mathrm{Ni}[7]$.

\section{High-energy behavior of fusion cross sections}

The cross sections we obtain in coupled-channels calculations are suppressed when compared to the no-coupling limit. This can be seen in Fig. 5] but the suppression looks small on a logarithmic plot. We therefore show a linear plot in Fig. 8 of the same cross sections for the two nickel isotopes. In both cases we see that the coupled-channels calculations that are based on the AW potential are suppressed at high energies when compared to the no-coupling limit. This is a well-known C. C. effect but the suppression is not always large enough to explain the measurements, in particular for heavy systems [10].

We also see in Fig. 8 that the C. C. results we obtain with the M3Y+repulsion potential are suppressed even further, and the suppression increases with increasing energy. This is naively what one would expect with shallower pockets at higher angular momenta. It is unfortunate that the data considered here do not reach very large cross sections, so it is difficult to assess the significance of the suppression we obtain. However, the trend is very encouraging because it helps explaining why the high-energy fusion data considered in Ref. [10] are suppressed so much compared to C. C. calculations and why it was necessary to use a large diffuseness of the ion-ion potential when fitting the data in the no-coupling limit. 


\section{INTERPRETATION OF THE RESULTS}

In the previous section we obtained an excellent fit to the fusion data for the two nickel isotopes in the C. C. calculations that were based on the M3Y+repulsion potential and in-going-wave boundary conditions. Here we discuss the significance of these results and the implications for other observables. We have in this connections also tested other types of repulsive cores, like the gaussian one of the type used in [32] but we arrived at the conclusion that they are inadequate in reproducing the fusion data we have investigated here. Thus there appears to be some limitations or constraints in constructing the shape of the ion-ion potential inside the barrier.

\section{A. Models of fusion}

The description of heavy-ion fusion is often based on the assumption that fusion occurs as soon as the Coulomb barrier has been penetrated. More precisely, it is expected that the fusion cross section obtained from the absorption in a short-range imaginary potential is essentially identical to the cross section obtained with in-going-wave boundary conditions (IWBC) [57]. Moreover, the calculated fusion cross section is expected to be insensitive to small variations in the radius where the absorption or IWBC are imposed.

In coupled-channels calculations, heavy-ion fusion is usually simulated by IWBC that are imposed at the minimum of the potential pocket, as for example in the computer code CCFULL [58] and also in the present work. The assumptions mentioned above have therefore rarely been challenged, and in cases where they have been tested, at energies close to the Coulomb barrier, they have usually passed the test. One exception is discussed in Ref. [59], where it was noticed that the calculated fusion cross section in one particular case showed an unacceptable variation with respect to the radius where the IWBC were imposed. This occurred in a case with particularly strong couplings. Another example is Ref. [55], where it was pointed out that certain unwanted oscillations in the cross sections, obtained at extreme subbarrier energies using the IWBC, could be suppressed by including a weak, short-range imaginary potential in the calculations.

In this work we try to demonstrate that the fusion at extreme subbarrier energies is sensitive not only to the thickness and the height of the Coulomb barrier but also to the minimum 


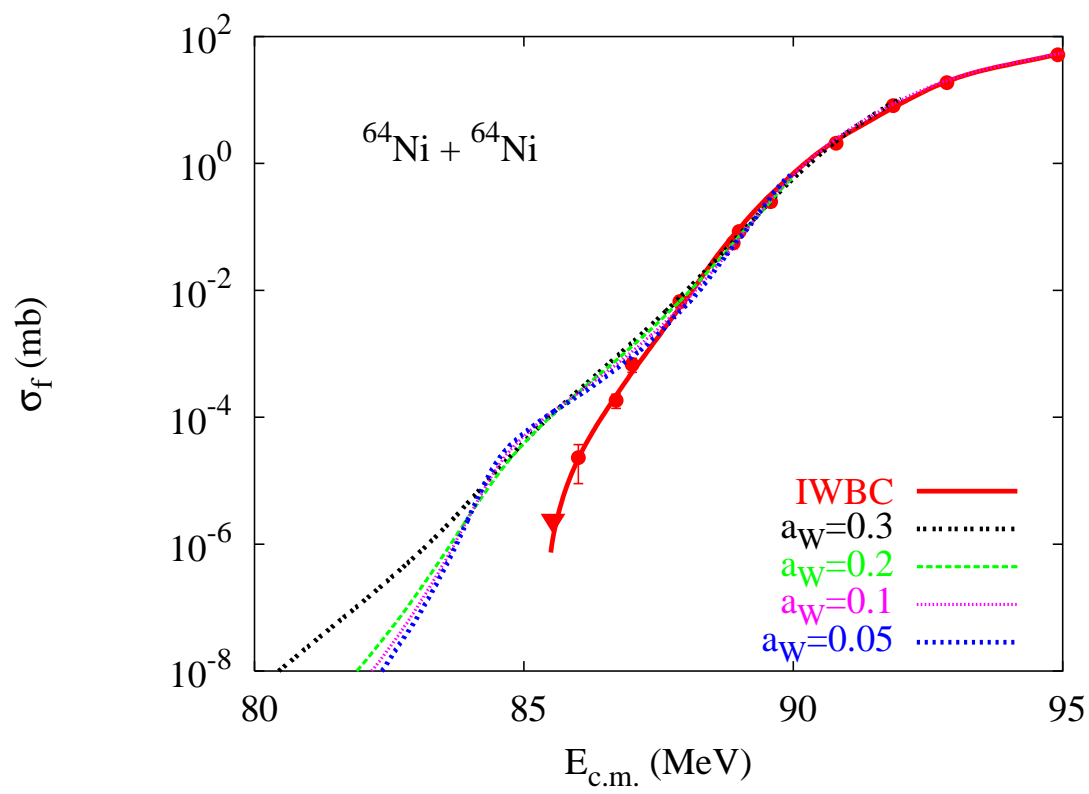

FIG. 9: (Color online). Dependence of the calculated fusion cross section (dashed curves) on the diffusenes $a_{W}$ of the imaginary potential. The results are compared to the data for ${ }^{64} \mathrm{Ni}+{ }^{64} \mathrm{Ni}[4]$ and the calculation, which is based on the IWBC without any absorption (the solid curve). The triangular data point at the lowest energy is an upper limit.

of potential pocket, where the IWBC are imposed. It is noted that the calculated fusion cross section, which is based on the IWBC, will vanish sharply as the energy approaches the minimum of the pocket. This sharp behavior is apparently what is needed to fit the data at extreme subbarrier energies, at least in the case of ${ }^{64} \mathrm{Ni}+{ }^{64} \mathrm{Ni}$.

We have also calculated the fusion of ${ }^{64} \mathrm{Ni}+{ }^{64} \mathrm{Ni}$ using, in addition to the M3Y+repulsion potential, a short-range imaginary potential of the form proposed in Eq. (3) of Ref. [55]. The results for different values of the diffuseness $a_{W}$ are shown in Fig. 9] by the dashed curves. They all make an excellent fit to the data above $10 \mu \mathrm{b}$ but they deviate significantly from the data at smaller cross sections. In contrast, the calculation discussed ealier (solid curve), which is based on the IWBC without any imaginary potential, is in perfect agreement with the data.

There is a simple reason why the dashed curves are enhanced compared to the solid curve (IWBC) in Fig. 9 at the lowest energies. First of all, the fusion obtained from the IWBC without an imaginary potential can only occur in the elastic channel at low energy, say below 87-88 MeV in the case considered here, and the cross section goes sharply to zero at the 
minimum of the pocket which is $85.4 \mathrm{MeV}$. The inelastic channels are energetically closed to fusion at these low energies and the associated wave functions are (exponentially) decaying at the boundary. When an imaginary potential is turned on, the fusion/absorption becomes possible through all channels. This explains qualitatively why the dashed curves are above the solid curve at low energy and why the conventional assumption about fusion described in the beginning of this subsection is not correct at extreme subbarrier energies.

\section{B. Average spin for fusion}

Another signature of a shallow pocket in the total ion-ion potential is a narrowing of the spin distribution for fusion as the center-of-mass energy decreases and approaches the pocket energy. In the past it has always been believed that the average angular momentum for fusion would approach a constant at low energy. This is the behavior that has been predicted by model calculations, including the C. C. [53], but it has not really been tested experimentally. An example is shown in Fig. 10, where the measurements of the $\gamma$-ray multiplicity from the compound nucleus formed in the fusion of ${ }^{64} \mathrm{Ni}+{ }^{64} \mathrm{Ni}$ have been converted into an average angular momentum for fusion [56]. The thin dashed curve shows the prediction based on the AW potential in the no-coupling limit (NOC $(\mathrm{AW})$ ). It approaches a constant value at low energy but the data are always above that limit. The solid curve in Fig. 10 shows the results we obtain in the C. C. calculations we discussed earlier, which were based on the $\mathrm{M} 3 \mathrm{Y}+$ repulsion potential. It is seen that these calculations predict a narrowing of the spin distribution as the energy approaches the pocket energy.

After completing our studies we realized that the low-energy behavior of several observables can have a strong sensitivity to the couplings to multi-phonon states. Although the fusion only occurs in the elastic channel at energies close to the minimum of the pocket, the polarization of inelastic channels can still have a large effect. We found, in particular, that couplings to the two-phonon octupole states are very important. This is illustrated in Fig. 10 by the dotted curve which was obtained without any couplings to the two-phonon octupole states. It is seen that the calculation in this case develops a rather sharp peak at $87.7 \mathrm{MeV}$. The peak disappears when the coupling to the two-phonon octupole states is included, as illustrated by the solid curve. Unfortunately, the data cannot tell us which of these two calculations is the most realistic. 


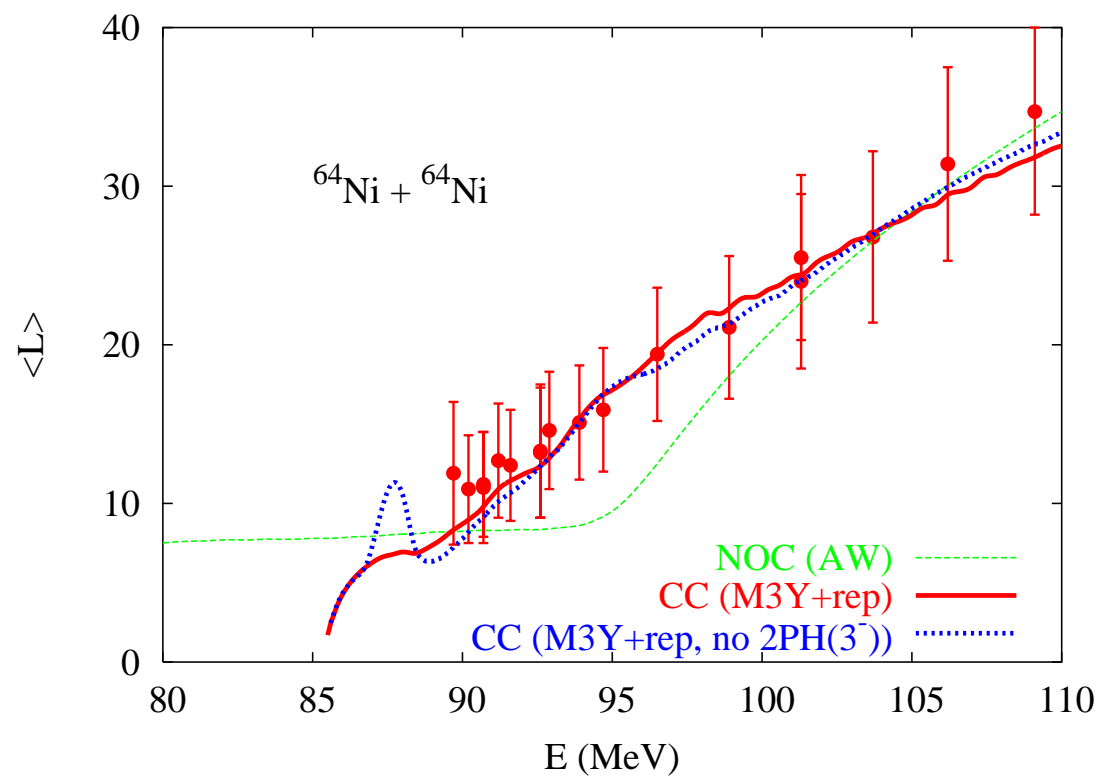

FIG. 10: (Color online). Average angular momentum for the fusion of ${ }^{64} \mathrm{Ni}+{ }^{64} \mathrm{Ni}$ obtained in C. C. calculations based on the M3Y+repulsion potential with (solid curve) and without (thick dotted curve) the effect of couplings to the two-phonon octupole states. The thin dashed curve was obtained in the no-coupling limit (NOC) using the AW potential. The data are from [56].

\section{Structures in calculated cross sections}

The local maximum that appears in the thick dotted curve in Fig. 10 at low energy is not a resonance. It is the result of a vanishing wave function in the elastic channel at the radial separation where the IWBC are imposed. When this condition is fulfilled at low energy, where fusion is restricted to the elastic channel, the fusion probability will vanish. When it occurs for a range of low angular momenta, it will result in a large average angular momentum for fusion and that explains the appearance of the peak in Fig. 10. The suppression of the fusion probability at low energy (which occurs when the two-phonon octupole states are not included in the calculation) can also produce a local minimum in the $S$-factor. This is illustrated by the dotted curve in Fig. 11, The solid curve, which includes the effect of couplings to the two-phonon octupole states, shows a single, broad maximum. In this particular case there is a clear preference for the solid curve which makes an excellent fit to the data.

In the calculations we presented for ${ }^{58} \mathrm{Ni}+{ }^{58} \mathrm{Ni}$ we did not include the two-phonon octupole 


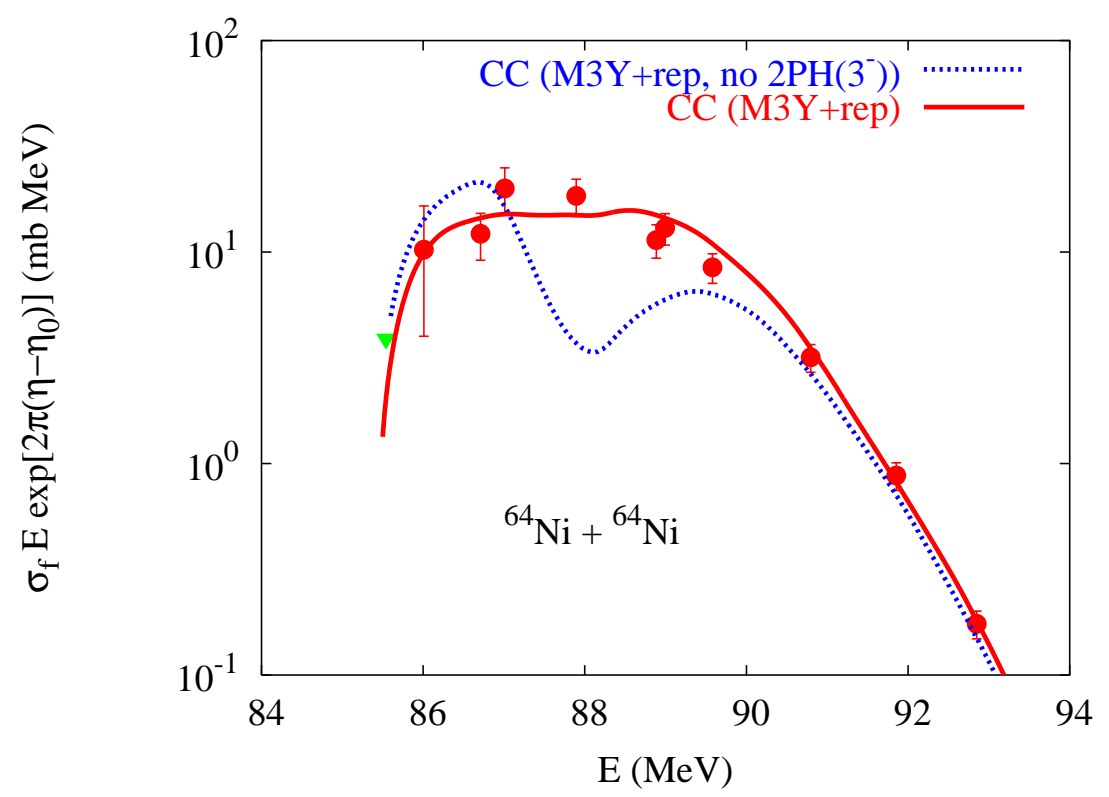

FIG. 11: (Color online). $S$ factors for the fusion of ${ }^{64} \mathrm{Ni}+{ }^{64} \mathrm{Ni}$ obtained in the C. C. calculations based on the M3Y+repulsion potential with (solid curve) and without (dotted curve) the effect of couplings to the two-phonon octupole states. The data are from Ref. [4]; the triangle is an upper limit.

states in the C. C. calculations because the excitation energy is very large, almost $9 \mathrm{MeV}$, so we did not expect these states would be important. In view of the above discussion it is now understandable why the calculated $S$-factor for ${ }^{58} \mathrm{Ni}+{ }^{58} \mathrm{Ni}$ shown in left panel of Fig. 6 develops a double-peaked structure at low energy. We have therefore repeated the calculations and included the two-phonon octupole states. The resulting $S$-factor exhibits a single, broad peak, just as we saw in the fusion of ${ }^{64} \mathrm{Ni}+{ }^{64} \mathrm{Ni}$.

It is not clear why the couplings to the two-phonon octupole states play such an important role as discussed above. However, the analysis of the ${ }^{64} \mathrm{Ni}+{ }^{64} \mathrm{Ni}$ data shows a perfect agreement with a single, broad $S$ factor peak, whereas the analysis of the existing fusion data for ${ }^{58} \mathrm{Ni}+{ }^{58} \mathrm{Ni}$ fusion has a strong preference for the double-peaked $S$-factor curve. In view of these findings it is of interest to continue the fusion measurements for ${ }^{58} \mathrm{Ni}+{ }^{58} \mathrm{Ni}$ to even lower energies because the existing data [7] shown in the left panel of Fig. [6 do not verify explicitly the double-peaked structure. 


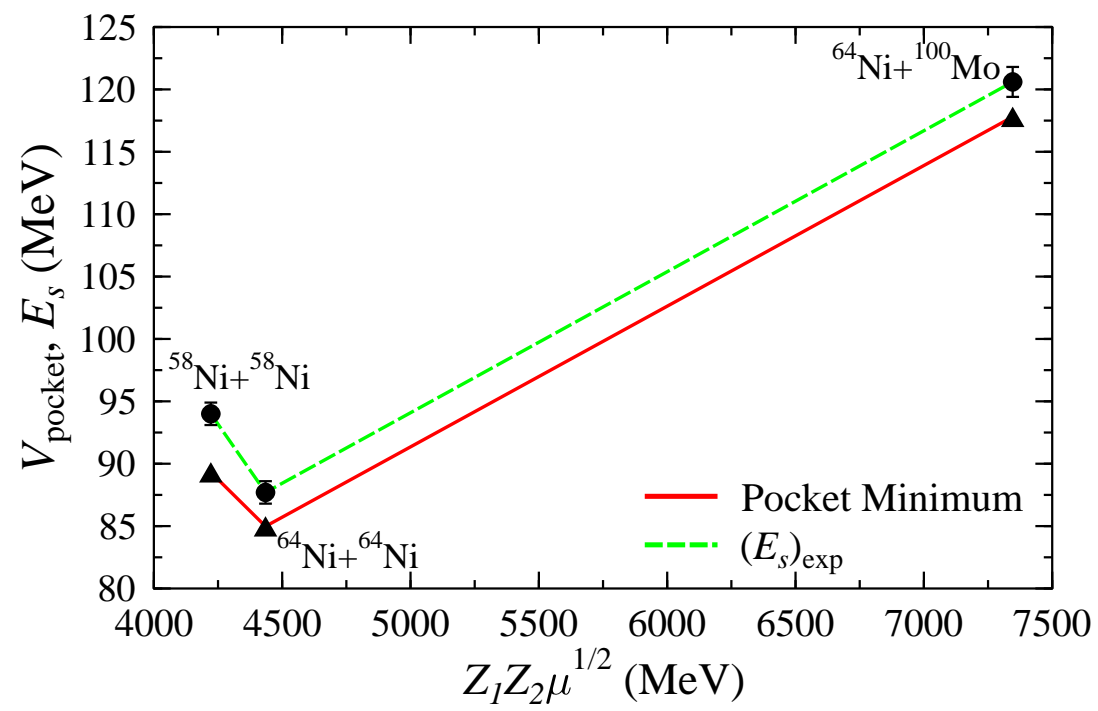

FIG. 12: (Color online). Plot of $V_{\text {pocket }}$ (triangles) and $E_{s}$ (solid points) versus $Z_{1} Z_{2} \sqrt{\mu}$ for the three reactions : ${ }^{58} \mathrm{Ni}+{ }^{58} \mathrm{Ni},{ }^{64} \mathrm{Ni}+{ }^{64} \mathrm{Ni},{ }^{64} \mathrm{Ni}+{ }^{100} \mathrm{Mo}$.

\section{Correlation between the pocket energy and $E_{s}$}

As we mentioned in the Introduction, Ref. [3] reported a correlation between the energy $E_{s}$ where the $S$-factor has a maximum and the parameter $Z_{1} Z_{2} \sqrt{\mu}$, with $\mu=A_{1} A_{2} /\left(A_{1}+A_{2}\right)$. In view of the idea of a shallow pocket advocated in the present paper we considered it useful to study the dependence of the minimum of the potential $V_{\text {pocket }}$, along with $E_{s}$, on the parameter $Z_{1} Z_{2} \sqrt{\mu}$. This is illustrated in Fig. 12 for the three cases we have investigated in this work. We note the pocket energy $V_{\text {pocket }}$ follows closely the energy $E_{s}$, where the $S$ has a maximum, the two results being separated by about 3 to $5 \mathrm{MeV}$.

\section{CONCLUSIONS}

We conclude that in order to explain the experimental fusion data at extreme subbarrier energies it is not necessary to adopt the hypothesis of an abnormal diffuseness of the internuclear potential as advocated in some publications. Such an assumption would be equivalent, within the folding model, to an unusual diffuseness of the target and projectile nuclear matter distributions. Proton diffusivities, as inferred from electron scattering measurements $\left({ }^{64} \mathrm{Ni}\right)$ or from HFB calculations $\left({ }^{58} \mathrm{Ni}\right.$ and $\left.{ }^{100} \mathrm{Mo}\right)$ are able to reproduce the 
excitation functions. Using a diffuseness of the neutron matter distribution in the range of 0.5-0.6 fm, which is a reasonable assumption for the moderately neutron rich nuclei considered in this paper, is necessary not only to realize a shallow pocket but also to obtain a good value for the height of the spherical Coulomb barrier. With these remarks we do not want to discard the appearance of a large diffuseness during the fusion process. Physically we expect an increase in the nuclear skin to appear in the neck (overlap) region but not over the entire surface of the reacting nuclei.

We have tried to advocate in this work that the understanding of the experimental data requires more than simply a modification in the curvature of the barrier (as used in the Hill-Wheeler approximation of the Wong formula), or the introduction of a simple recipe for the repulsive potential like a gaussian. We used the double-folding potential that is based on the Reid parametrization of the M3Y interaction, and realistic parameters of the proton and neutron distributions of both target and projectile. We have supplemented this potential with a repulsive potential that takes into account the incompressibility of the nuclear matter.

We also conclude that it is necessary to define the fusion in terms of in-going-wave boundary conditions. When these consitions are imposed at the minimum of the potential pocket, it is possible to reproduce the steep energy dependence of the fusion data at extreme subbarrier energies. Simulating the fusion by the absorption in an imaginary potential, on the other hand, does not allow us to reproduce the steep falloff of data at the lowest energies.

In the two cases that recently were measured at ATLAS, namely, ${ }^{64} \mathrm{Ni}+{ }^{64} \mathrm{Ni}$ and ${ }^{64} \mathrm{Ni}+{ }^{100} \mathrm{Mo}$, our calculations show a single broad maximum in the $S$-factor at the lowest measured energies in agreement with the measurements. In contrast, our calculations for ${ }^{58} \mathrm{Ni}+{ }^{58} \mathrm{Ni}$ show a double-peaked structure of the $S$-factor. We find that the predicted shape of the low-energy $S$-factor is very sensitive to the couplings to the two-phonon octupole states. Thus if we ignore these couplings, the $S$-factor develops a double-peaked structure at low energy, whereas a strong coupling to the these states (as in a harmonic vibration) tends to produce a single, broad low-energy peak. It is therefore important to test the predicted $S$-factor experimentally at lower energies, in particular in the case of ${ }^{58} \mathrm{Ni}+{ }^{58} \mathrm{Ni}$.

We noticed for all three fusion reactions studied in this paper a nice correlation between the minimum of the potential pocket $V_{\min }$ and the experimentally extracted reference energy $E_{s}$, where the $S$-factor reaches a maximum. A systematic study of fusion reactions over a wider range of values of the parameter $Z_{1} Z_{2} \sqrt{\mu}$ is necessary to confirm this apparent 
correlation, and the conjecture of a repulsive core for overlapping configurations. We also mentioned another possible signature of a shallow pocket, which is the narrowing of the spin distribution at energies below $E_{s}$. This is what our C. C. calculations predict and it can be tested by measurements of the $\gamma$-ray multiplicity emitted from the compound nucleus.

Before submitting this paper further evidence on the hindrance in the sub-barrier fusion of ${ }^{48} \mathrm{Ca}+{ }^{96} \mathrm{Zr}$ was reported [60]. This conclusion resulted from the analysis of the logarithmic derivative which exhibits a steep increase at the lowest measured cross sections.

\section{Acknowledgements}

One of the authors (Ş.M.) is grateful to the Fulbright Commission for financial support and for the hospitality of the Physics Division at Argonne National Laboratory where this work was completed. H.E. acknowledge the support of the U.S. Department of Energy, Office of Nuclear Physics, under Contract No. DE-AC02-06CH11357. We are also grateful to C. L. Jiang, B. B. Back, R. V. F. Janssens and K. E. Rehm for constructive comments on this article.

[1] Ş. Mişicu and H. Esbensen, Phys. Rev. Lett. 96, 112701 (2006).

[2] C. L. Jiang et al., Phys. Rev. Lett. 89, 052701 (2002).

[3] C. L. Jiang, H. Esbensen, B. B. Back, R. V. F. Janssens, and K. E. Rehm, Phys. Rev. C 69, 014604 (2004).

[4] C. L. Jiang et al., Phys. Rev. Lett. 93, 012701 (2004).

[5] C. L. Jiang et al., Phys. Rev. C 71, 044613 (2005).

[6] C. L. Jiang, B. B. Back, H. Esbensen, R. V. F. Janssens, and K. E. Rehm, Phys. Rev. C 73, 014613 (2006).

[7] M. Beckerman, M. Salomaa, A. Sperduto, J. D. Molitoris and A. Di Rienzo, Phys. Rev. C 25, 837 (1982).

[8] J. G. Keller et al., Nucl. Phys. A452, 173 (1986).

[9] K. Hagino, N. Rowley and M. Dasgupta, Phys. Rev. C 67, 054603 (2003). 
[10] J. O. Newton, R. D. Butt, M. Dasgupta, D. J. Hinde, I. I. Gontchar, C. R. Morton and K. Hagino, Phys. Rev. C 70, 024605 (2004).

[11] C. H. Dasso and G. Pollarolo, Phys. Rev. C 68, 054604 (2003);

G. Pollarolo, Prog. Theor. Phys. (Kyoto), Suppl.154, 201 (2004).

[12] R. A. Broglia and Aage Winther, Heavy Ion Reactions, Lecture Notes, Volume I: The Elementary Processes, (Addison-Wesley Pub. Co., MA, 1991), p. 114, Eqs. (40-41,44-45).

[13] Ö. Akyüz and A. Winther, Proc. of the Enrico Fermi School of Physics, 1979, course on Nuclear Structure and Heavy-ion Reactions, eds. R. A. Broglia, C. H. Dasso, and R. Ricci (North Holland, Amsterdam, 1981).

[14] W. M. Seif, J. Phys. G30, 1231 (2004).

[15] H. Esbensen and S. Landowne, Phys. Rev. C 35, 2090 (1987).

[16] K. Hagino, N. Takigawa, M. Dasgupta, D. J. Hinde, and J. R. Leigh, Phys. Rev. C 55, 276 (1997).

[17] C. J. Lin, Prog. Theor. Phys. (Kyoto), Suppl. 154, 184 (2004).

[18] B.G. Giraud, S. Karataglidis, K. Amos and B. A. Robson, Phys. Rev. C 69, 064613 (2004).

[19] A. O. Caldeira and A. J. Leggett, Phys. Rev. Lett. 48, 1571 (1982).

[20] E. M. Burbidge, G. R. Burbidge, W. A. Fowler and F. Hoyle, Rev. Mod. Phys. 29, 547 (1957).

[21] K. A. Erb and D. A. Bromley, in Treatise in Heavy-Ion Science, Vol. 3, ed. D. A. Bromley (Plenum, 1985).

[22] L. Barrón-Palos et al., Nucl. Phys. A779, 318 (2006).

[23] J. R. Patterson, B. N. Nagorčka, G. D. Symons, W. M. Zuk, Nucl. Phys. A165, 545 (1971).

[24] D. A. Bromley, J. A. Kuehner and E. Almqvist, Phys. Rev. Lett. 4, 365 (1960).

[25] W. Scheid, W. Greiner and R. Lemmer, Phys. Rev. Lett. 25, 176 (1970).

[26] R. Zurmühle, P. Kutt, R. R. Betts, S. Saini, F. Haas, and Ole Hansen, Phys. Lett. B129, 384 (1984).

[27] R. R. Betts, B. B. Back, and B. G. Glagola, Phys. Rev. Lett. 47, 23 (1981).

[28] H. J. Rose and G. A. Jones, Nature 307, 245 (1984).

[29] J. H. Hamilton et al., J. Phys. G20, L85 (1994).

[30] A. Krasznahorkay et al., Proceedings of the Symposium on Nuclear Clusters, W. Scheid and R. Jolos eds., EP Systema, Debreczen (2002).

[31] R. J. Munn, B. Block and F. B. Malik, Phys. Rev. Lett. 21, 159 (1968). 
[32] G. Michaud, Phys. Rev. C 8, 525 (1973).

[33] G. R. Satchler and W. G. Love, Phys. Rep. 55, 183 (1979).

[34] M. Seiwert, W. Greiner, V. Oberacker and M. J. Rhoades-Brown, Phys. Rev. C 29477 (1984).

[35] M. Seiwert, Untersuchung des Kernpotentials in der Massregion überkritischer Systeme mittels phänomenologischer Modelle, Disertation, J. W. Goethe Universität, Frankfurt am Main (1985); M. Seiwert, W. Greiner, and W. T. Pinkston, J. Phys. G11, L21 (1985).

[36] Ş. Mişicu and D. Protopopescu, Acta Phys. Pol. B30, 127 (1999).

[37] M. E. Brandan and G. R. Satchler, Phys. Rep. 285, 143 (1997).

[38] Dao T. Khoa, G. R. Satchler and W. von Oertzen, Phys. Rev. C 56, 954 (1997).

[39] I. I. Gontchar, D. J. Hinde, M. Dasgupta, and J. O. Newton, Phys. Rev. C 69, 024610 (2004).

[40] B. Block and F. B. Malik, Phys. Rev. Lett. 19, 239 (1967).

[41] W. Scheid, R. Ligensa and W. Greiner, Phys. Rev. Lett. 21, 1479 (1968).

[42] V. Yu. Denisov, Phys. Lett. B526, 315 (2002).

[43] J. Blocki, J. Randrup, W. J. Swiatecki and C. F. Tsang, Ann. Phys. (N.Y.) 105, 427 (1977).

[44] W. D. Myers and W. J. Swiatecki, Phys. Rev. C 62, 044610 (2000).

[45] Ş. Mişicu and W. Greiner, Phys. Rev. C 69, 054601 (2004).

[46] W. D. Myers and W. J. Swiatecki, Phys. Rev. C 57, 3020 (1998).

[47] J. Eisenberg and W. Greiner, Nuclear Theory, Vol. I, Phenomenological Models, (NorthHolland, Amsterdam 1988).

[48] E. Uegaki and Y. Abe, Prog. Theor. Phys., 90, 615 (1993).

[49] G. Bertsch, W. Borysowicz, H. McManus and W. G. Love, Nucl. Phys. A284, 399 (1977).

[50] A. Sandulescu, Ş. Mişicu, F. Carstoiu and W. Greiner, Elem. Chast. At. Yadra 30, no. 4, 908-953 (1999) \& Phys. Part. Nucl. 30, 386 (1999).

[51] H. De Vries, C. W. Jaeger and C. De Vries, At. Data and Nucl. Data Tables, 36, 495 (1987).

[52] S. Goriely, M. Samyn, P.-H. Heenen, J. M. Pearson and F. Tondeur, Phys. Rev. C 66, 024326 (2002). See also http://www-nds.iaea.org/RIPL-2/

[53] H. Esbensen, Prog. Theor. Phys. (Kyoto), Suppl. 154, 11 (2004).

[54] Ş. Mişicu and W. Greiner, Phys. Rev. C 66, 044606 (2002).

[55] H. Esbensen, Phys. Rev. C 72, 054607 (2005).

[56] D. Ackerman et al., Nucl. Phys. A609, 91 (1996).

[57] S. Landowne and Steven C. Pieper, Phys. Rev. C 29, 1352 (1984). 
[58] K. Hagino, N. Rowley, A.T. Kruppa, Comput. Phys. Commun. 123, 143 (1999).

[59] S. Landowne, Steven C. Pieper, and F. Videbaek, Phys. Rev. C 35, 597 (1987).

[60] A. M. Stefanini et al., Phys. Rev. C 73, 034606 (2006). 